\title{
Magnetic anisotropy and evolution of ground-state domain structures in bcc $\mathrm{Fe}_{81} \mathrm{Ni}_{19} / \mathrm{Co}(001)$ superlattices
}

\author{
R. Bručas, ${ }^{1}$ H. Hafermann, ${ }^{2}$ I. L. Soroka, ${ }^{3}$ D. Iuşan, ${ }^{1}$ B. Sanyal, ${ }^{1}$ M. I. Katsnelson, ${ }^{4}$ O. Eriksson, ${ }^{1}$ and B. Hjörvarsson ${ }^{1}$ \\ ${ }^{1}$ Department of Physics, Uppsala University, Box 530, 75121 Uppsala, Sweden \\ ${ }^{2}$ I. Institute of Theoretical Physics, University of Hamburg, 20355 Hamburg, Germany \\ ${ }^{3}$ Department of Materials Chemistry, Uppsala University, Box 576, 75123 Uppsala, Sweden \\ ${ }^{4}$ Institute for Molecules and Materials, Radboud University, 6525 ED Nijmegen, The Netherlands \\ (Received 22 January 2008; revised manuscript received 19 May 2008; published 18 July 2008)
}

\begin{abstract}
The magnetic anisotropy and evolution of striped magnetic domain structures in bcc $\mathrm{Fe}_{81} \mathrm{Ni}_{19} / \mathrm{Co}(001)$ superlattices with the total thickness ranging from 85 to $1370 \mathrm{~nm}$ has been studied by magneto-optical Kerr effect and magnetic force microscopy. At a thickness of about $85 \mathrm{~nm}$ [25 bilayers (BL)] the domains appear as stripe domains, typical for perpendicular anisotropy films, with the weak cubic anisotropy of the in-plane magnetization component stabilizing the stripe direction. The magnetic domain period strongly depends on the thickness of the superlattice. As the thickness increases, the equilibrium magnetization orients at oblique angles with respect to the film plane and continuously varies with the thickness from in-plane to out-of-plane. We first apply a simple phenomenological model which correctly predicts the transition from in-plane to out-of-plane magnetization as well as increasing domain period and saturation field with increasing BL number. The results indicate the presence of partial flux-closure domains at the film surface with the tilt angle continuously varying with the superlattice thickness. By solving a linearized Landau-Lifshitz equation together with Maxwell's equations in magnetostatic approximation for samples consisting of up to 1000 individual layers, we calculate the spin-wave dispersion and determine the stability conditions for the saturated ferromagnetic state. From these results the dependence of the saturation field on the number of layers is inferred and agrees well with the experiment. The uniaxial bulk anisotropy is attributed to distortions along the $c$ axis and the results further show evidence for the presence of an easy-plane interface anisotropy in these samples.
\end{abstract}

DOI: 10.1103/PhysRevB.78.024421

PACS number(s): 75.60.- d, 75.70.-i, 75.75.+a

\section{INTRODUCTION}

Symmetry breaking, e.g., at interfaces and surfaces, and proximity effects strongly influence the ground-state magnetic properties in artificial heterostructures. The competition of short-range exchange and long-range dipolar interactions can lead to a wide variety of ordering effects and has been extensively studied both theoretically ${ }^{1-3}$ and experimentally. ${ }^{4-11}$ The research is to a large extent driven by the interest in better understanding of magnetic anisotropies, in particular, the tendency of the easy magnetization axis to be oriented perpendicular to the film plane and related domain structure formation.

Magnetic anisotropies originate from two different sources: The shape anisotropy is caused by the long-ranged dipolar interaction while magnetocrystalline anisotropies stem from relativistic spin-orbit coupling. Due to the spinorbit coupling, the magnetization couples to the lattice and thus gives rise to an additional energy term which reveals the symmetry of the crystal structure. In cubic crystals the corresponding anisotropy energy thus has cubic symmetry. However, if the cubic symmetry is slightly broken due to lattice distortions, this may give rise to additional uniaxial anisotropy contributions.

Theoretical calculations based on continuum approximations ${ }^{12-14}$ in perpendicular anisotropy films predict the ground state of out-of-plane anisotropy films to consist of stripes with up and down spins if an exchange interaction exceeds some small critical value and to be always stable with respect to the homogeneous ferromagnetic state. ${ }^{3}$ The magnetic striped phases are typically expected in micrometer thick films ${ }^{12,15}$ as a result of the competition between the exchange interactions, which favor parallel alignment of neighboring spins, and much weaker but long-range dipolar coupling, which favors antiparallel alignment over larger distances. ${ }^{12}$ The theory for such a case was developed first by Kitte $^{16}$ in the 1950s and is valid for film thicknesses which are large enough compared to the domain size, the latter being proportional to the square root of the film thickness.

Since the discovery of perpendicular magnetic anisotropy (PMA) in Co-based systems, substantial effort has been put into studies of metallic multilayers and superlattices ${ }^{1,5-11,17-20}$ due to possible applications as magnetooptic recording media. Surface and interface anisotropies can cause perpendicular magnetization with respect to the film plane and have been identified as the source of the PMA in some Co-based heterostructures. ${ }^{21-23}$ Shape anisotropies due to dipolar interaction tend to orient the magnetization into the film plane. With increasing film thickness, the dipolar interactions gain relative importance and can cause the spinreorientation transition, ${ }^{9}$ as, e.g., was observed in ultrathin $\mathrm{Fe}-\mathrm{Co}$ films ${ }^{24}$ or ultrathin $\mathrm{Fe}$ films on $\mathrm{Cu}^{25}$ On the contrary, out-of-plane magnetization can be obtained by further increasing the film thickness if an out-of-plane bulk anisotropy is present, where the transition is driven by the competition between magnetocrystalline and shape anisotropies. In the situations where competing bulk and interface anisotropies are present, the spin-reorientation transition is driven by varying the interface density.

Thus, the interplay between shape and bulk anisotropies and surface or interface induced anisotropies, i.e., thickness- 
dependent and surface-interface controlled properties, opens new ways to control and manipulate the properties of future data storage devices.

In the present work we study the nature of the magnetic anisotropies, the interplay between them and the effect of the shape anisotropy on the evolution of the ground-state domain structure in bcc $\mathrm{Fe}_{81} \mathrm{Ni}_{19} / \mathrm{Co}(001)$ superlattices grown on $\mathrm{MgO}(001)$ substrates in the thickness range from 85 to 1370 nm.

\section{EXPERIMENT}

A detailed description of the growth conditions and structural properties of the bcc $\mathrm{Fe}_{81} \mathrm{Ni}_{19} / \mathrm{Co}(001)$ superlattices is given elsewhere, ${ }^{26}$ so only the important details are presented here.

$\mathrm{Fe}_{81} \mathrm{Ni}_{19} /$ Co superlattices were grown onto an $\mathrm{MgO}(100)$ single crystal substrate using dc magnetron sputtering. The thickness of the layers was fixed at $1.72 \mathrm{~nm}$ for the FeNi and $1.70 \mathrm{~nm}$ for the Co layers and the number of repetitions was varied in the range from 25 to 400 bilayers (BL), where $1 \mathrm{BL}$ $\sim 3.42 \mathrm{~nm}$, corresponding to a range of 85 to $1370 \mathrm{~nm}$ in the total thickness. All superlattices were grown at $170{ }^{\circ} \mathrm{C}$ and the deposition rates were 0.035 and $0.08 \mathrm{~nm} / \mathrm{s}$ for $\mathrm{FeNi}$ and Co, respectively. These values were obtained in situ by a quartz microbalance and then calibrated with the thickness measurements using low angle $\left(2 \Theta=1-15^{\circ}\right)$ x-ray reflectivity, which gave the individual layer spacing, the total thickness, and the bilayer period of the multilayer structure. All the samples showed well-developed superlattice peaks up to third order indicating a distinct compositional modulation along the film growth direction. Simulations of the reflectivity data performed using WINGIXA ${ }^{27}$ were consistent with a $1.5 \pm 0.5$ monolayer $(\mathrm{ML})$ thickness variation and roughness of the layers.

The crystallographic structure of the superlattices was investigated by high angle $(2 \Theta=20-100)$ x-ray diffraction using a Siemens D5000 diffractometer, which confirms good quality of the superlattices (not shown here). Only one peak of (002) reflection from the bcc $\mathrm{Fe}_{81} \mathrm{Ni}_{19}$ / Co lattice surrounded by two first order and two second-order satellites was found in all the samples. The full width at half maximum (FWHM) of the rocking curves of the (002) reflection varied from $0.2^{\circ}$ to $0.5^{\circ}$ depending on the BL number in the superlattice, indicating well-oriented, single-crystal structures. The superlattices do not grow coherently with the substrate due to large $(4.6 \%)$ lattice mismatch between the film and the substrate. Furthermore, the resulting films have a slight in-plane orthorhombic distortion which makes the [110] and [1 $\overline{1} 0]$ directions nonequivalent. It was found previously ${ }^{28}$ that such an orthorhombic distortion causes a

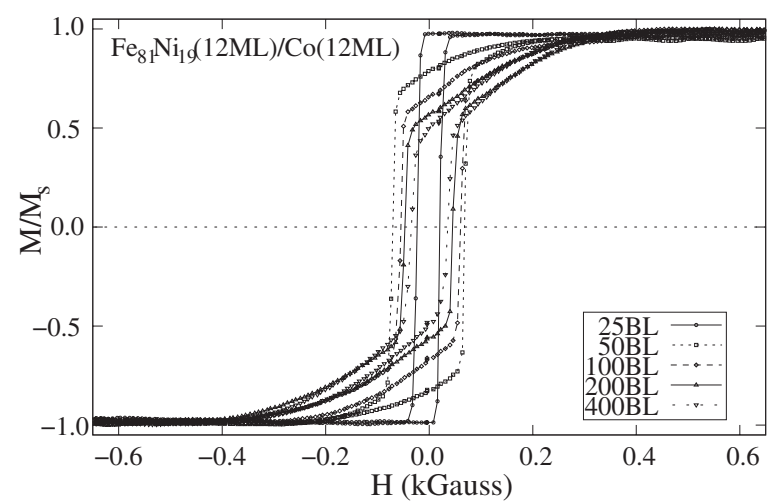

FIG. 1. Room-temperature magnetization hysteresis loops (normalized to the saturation magnetization $M_{S}$ ) of the $\mathrm{Fe}_{81} \mathrm{Ni}_{19} / \mathrm{Co}$ superlattices for different numbers of bilayers in the range from 25 to $400 \mathrm{BL}$, measured with a magnetic field applied in the film plane.

weak uniaxial in-plane magnetic anisotropy and will be discussed below in more details.

Magnetization hysteresis $(M-H)$ loop measurements for the superlattices were performed at room temperature by using the magneto-optical Kerr effect (MOKE) in the longitudinal geometry with $s$-polarized light. The experimental setup allows rotation of the sample around its surface normal making the measurements of $M-H$ curves in arbitrary inplane directions possible.

We first investigate the orientation of the magnetization direction depending on the thickness (number of BL) of the superlattices starting from the thinnest $(25 \mathrm{BL})$ sample where the stripe domains have been observed. Figure 1 shows typical MOKE hysteresis loops measured at room temperature with the applied field parallel to the film plane. For the superlattices consisting of 50-400 BL the in-plane remanence is smaller than unity showing that the easy axis of magnetization lies out-of-plane. The oblique orientation of the magnetization with respect to the film surface was confirmed by the magnetic force microscopy (MFM) images, as discussed below. The remanence decreases as the film thickness is increased so that the tilt angle of the magnetization vector varies with the BL number. This looks rather natural since the magnetostatic (demagnetizing) energy varies with the thickness of the film.

In order to examine the qualitative behavior of the saturation field, we roughly estimated the values from the hysteresis curves in Fig. 1. To this end, the hysteresis loops were normalized to their maximal value, and the field where the magnetization reaches 1 was taken as an estimate for the saturation field. The values are given in Table I. The magnetization of the $25 \mathrm{BL}$ superlattice is almost fully oriented in-plane indicating that the sample is close to the transition to the homogeneously magnetized state. For small BL numbers the saturation field varies more rapidly while it saturates

TABLE I. Experimental values of the saturation field estimated from the MOKE hysteresis curves in Fig. 1. The actual saturation fields are expected to be larger, as explained in the text.

\begin{tabular}{cccccc}
\hline \hline BL & 25 & 50 & 100 & 200 & 400 \\
$H_{s}$ (kGauss) & 0.0 & 0.25 & 0.45 & 0.6 & 0.6 \\
\hline \hline
\end{tabular}



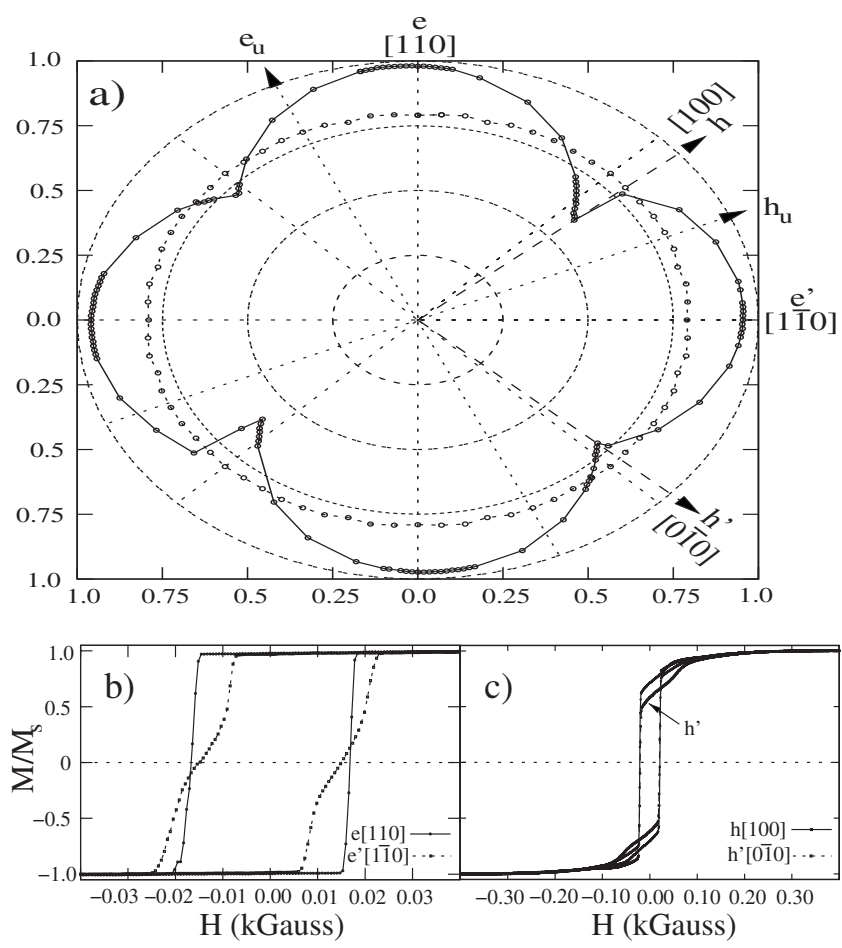

FIG. 2. Room-temperature magnetization data for $\mathrm{Fe}_{81} \mathrm{Ni}_{19} / \mathrm{Co}$ superlattices consisting of 25 and 50 BL. (a) The remanent magnetization as a function of the in-plane rotation angle for the superlattices with 25 (squares) and 50 (circles) BL repetitions. The crystallographic directions are marked with pointers and the dashed lines show the tilt of the hard $(h)$ axes. The spacing between data points in this region is $1^{\circ}$. Magnetization hysteresis loops for two easy directions [110] and [1 $\overline{10}]$ and the hard [100] and [0 $\overline{10}]$ directions are presented in (b) and (c), respectively.

for larger BL numbers. It is important to note that the true saturation fields for these samples are expected to be higher than those obtained from the MOKE measurements, since this is a surface sensitive technique (the typical MOKE penetration depth is about $60 \AA$ ).

In Fig. 2(a) we show the values of the normalized remanent magnetization as a function of in-plane rotation angles for the superlattices consisting of 25 and $50 \mathrm{BL}$. As seen in the figure, the superlattice consisting of 25 BL exhibits a fourfold in-plane rotational anisotropy with an easy axis $(e)$ in the [110] and a hard axis $(h)$ in the [100] directions. A clear difference in the magnetization values can be seen between the two hard [100] and [010] directions. For the perfect cubic crystal, the hard axes would coincide with the high-symmetry $[100]([0 \overline{1} 0])$ directions, but we find the position of the hard axes (defined as the local minimum of the in-plane remanence) to be tilted by an angle of approximately $4^{\circ}$ from these directions. The easy [110] and [1 $\left.\overline{1} 0\right]$ directions are also found to be nonequivalent as can be clearly seen from Fig. 2(b). The difference of magnetic response to [110] and [1 $\overline{1} 0]$ directions, which are magnetically equivalent for the perfect fourfold symmetric cubic crystal, can be explained by the presence of a superimposed uniaxial in-plane anisotropy. The easy axis of this uniaxial anisotropy $\left(e_{u}\right)$ lies between the [010] and [110] directions, making the

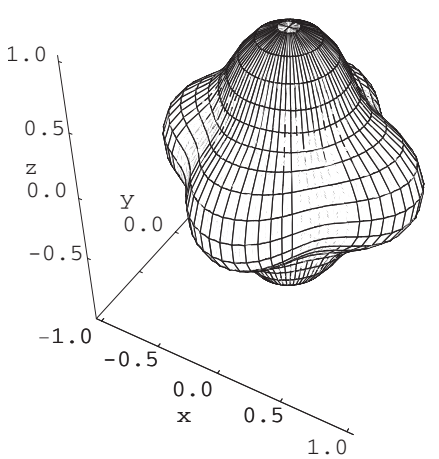

FIG. 3. Constant energy surface for the cubic anisotropy energy functional, Eq. (1) for negative $K_{c}$ as a function of the direction cosines of the magnetization. For oblique orientations of the magnetization with respect to the film surface, the anisotropy functional is approximately rotationally invariant.

easy directions $\left(e\right.$ and $\left.e^{\prime}\right)$ nonequivalent. The easy axis of the uniaxial anisotropy $\left(e_{u}\right)$ lies closer to $(e)$, so that this is the easy axis and $\left(e^{\prime}\right)$ is an intermediate axis. The hysteresis curve measured along the intermediate axis shows the corresponding two-step magnetization reversal via the easy axis. The weak in-plane uniaxial anisotropy is a consequence of the slightly broken cubic symmetry and has its origin in the orthorhombic distortion of the crystal, as observed in XRD measurements. In-plane uniaxial anisotropy has also been observed previously in Fe (Ref. 29) and Co (Refs. 30 and 31) ultrathin films induced by roughness and periodical step arrangement of the substrate.

Figure 2(a) further shows that the maximal remanence value for the superlattice with $25 \mathrm{BL}$ is slightly less than 1 ( 0.98 for the $e$ and 0.95 for the $e^{\prime}$ ), indicating the presence of a weak out-of-plane component of the magnetization. This fact was later confirmed by the MFM measurements (to be analyzed below) which showed the existence of stripe domains in this sample. No change in the remanence could be detected by varying the in-plane applied field angle for the superlattice with $50 \mathrm{BL}$. The remanence of about 0.8 for all in-plane directions indicates the presence of a strong out-ofplane magnetization component and a "rotatable" anisotropy depending on the previous saturation direction, while the inplane anisotropy is not detectable. The same results were obtained for the superlattices containing 100, 200, and 400 BL. The reason for this observation is nicely illustrated by plotting the energy surface for a cubic anisotropy of the form

$$
E_{c}=K_{c}\left(m_{x}^{2} m_{y}^{2}+m_{x}^{2} m_{z}^{2}+m_{y}^{2} m_{z}^{2}\right),
$$

for negative $K_{c}{ }^{32}$ Figure 3 shows that for small angles of the magnetization with respect to the surface normal (i.e., for sufficiently large $m_{z}$ ), the energy is almost independent of the in-plane angle. This is consistent with the observations for the $50 \mathrm{BL}$ and thicker samples. It should be noted that for $K_{c}<0$, the [111] directions are the corresponding easy directions. However, since one measures the projections onto the film plane, one finds the easy directions along [110].

The imaging of the magnetic domains was performed at room temperature in zero field with a MFM (digital instruments, DI 3100), operated in tapping lift mode. This mode 
allows simultaneous acquisition and clear separation between the topographic and magnetic data. Various types of commercially available tips with low (FeCoNi coating) and high coercivity ( $\mathrm{CoCr}$ coating) were tested before imaging. All tips were magnetized vertically with a permanent magnet before imaging. It was confirmed that the domain period extracted from MFM images measured with both types of tips was essentially the same. The MFM image contrast is proportional to the gradient of the magnetic force between tip and sample. In order to elucidate the tip influence on the domain structure the tip-to-sample distance was varied in the range from 50 to $150 \mathrm{~nm}$ during magnetic imaging. The average domain period and the angular distribution were determined by a two-dimensional Fourier transform algorithm (2DFT).

The MFM images for the superlattices in the as-grown (virgin) and in-plane remanent states (after in-plane magnetic saturation) varying the BL number are shown in Figs. 4(a)-4(j). The images clearly show well-ordered magnetic domain patterns with alternating up and down magnetizations. Such stripe domain structures in ferromagnetic materials arise from the balance between the magnetocrystalline, dipolar, and exchange energies. The magnetization in alternate domains is oriented in opposite directions and is therefore separated by Bloch walls the width of which is determined by a balance between the magnetocrystalline and exchange energies. ${ }^{33}$

The observed magnetic up and down states originate from magnetic-flux lines on the surface of the sample. The formation of partial flux-closure domains inside the sample near the surface together with the magnetization within the domain walls creates a weak in-plane magnetization which might be responsible for the high degree of order (stripe direction) in the virgin state. It was observed that the stripe direction in the virgin state always points along the edge of the sample which corresponds to the easiest $(e)$ magnetization axis for the $25 \mathrm{BL}$ superlattice. As it was shown above (see Fig. 2), the cubic anisotropy, being relatively strong in the $25 \mathrm{BL}$ superlattice, is not detectable for larger BL numbers and out-of-plane magnetization, but is still present in these samples and favors alignment of the in-plane magnetization along this direction.

Flux-closure domains with in-plane magnetization are impossible to resolve since the MFM probes only the magnetic stray fields outside the sample and does not provide any information about the magnetic profile within the film. However, the existence of these domains is consistent with the fact that the average magnetization is oriented at oblique angles with respect to the film surface, indicating an intermediate value of the quality factor $Q$ (the ratio of anisotropy to demagnetization energy), commonly used to characterize the stripe domain structures. ${ }^{33}$ For small $Q(Q \ll 1)$, the magnetostatic energy dominates over the anisotropy energy and it is energetically favorable to maintain in-plane magnetization. For large $Q(Q \gg 1)$, the anisotropy energy dominates, favoring stripe domain formation with perpendicular magnetization relative to the film surface, which leads to surface magnetic charges. At intermediate $Q$ values $(Q \simeq 1)$, the stripe domains with Bloch-like walls in the middle of the sample and partial flux closures at the top and bottom of the film are

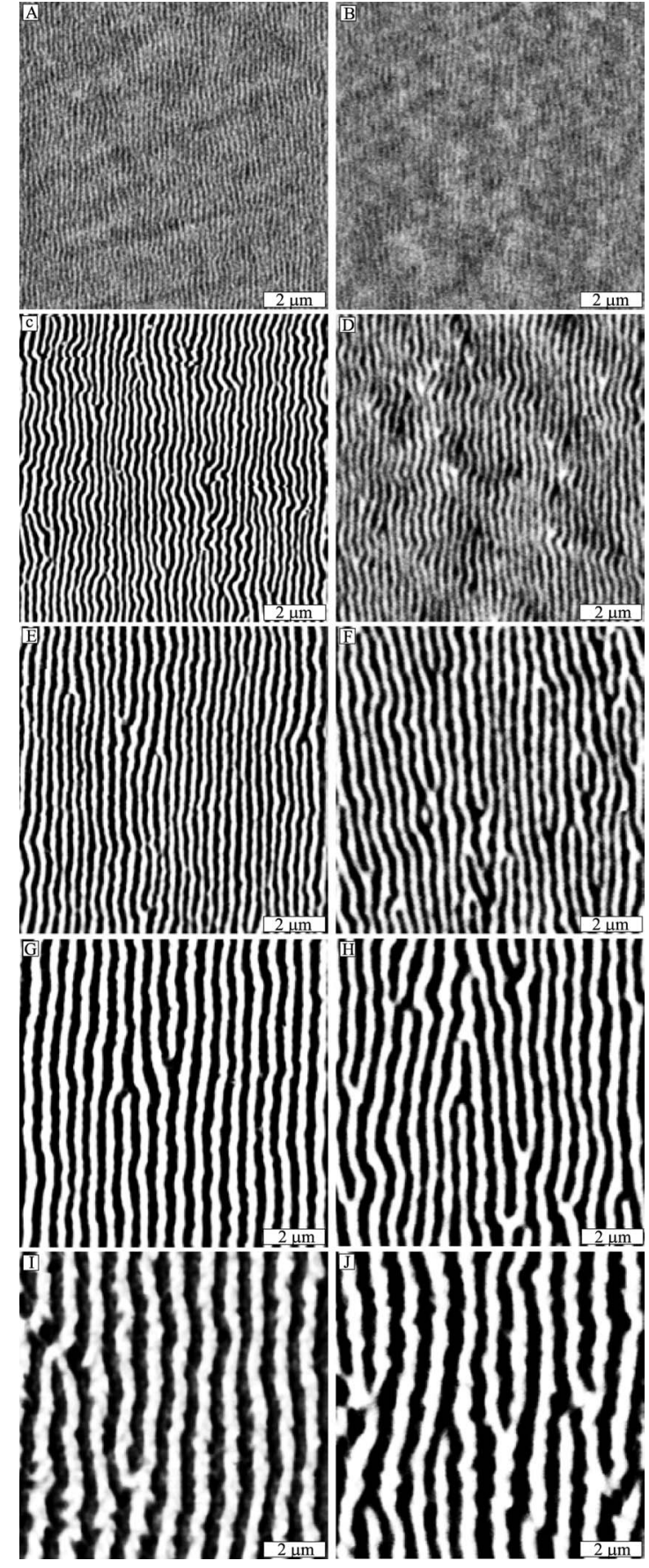

FIG. 4. The MFM images obtained in zero applied field of bcc $\mathrm{Fe}_{81} \mathrm{Ni}_{19} / \mathrm{Co}$ superlattices in the as-deposited (virgin) state for (a) $25 \mathrm{BL}$, (c) $50 \mathrm{BL}$, (e) $100 \mathrm{BL}$, (g) $200 \mathrm{BL}$, and (i) $400 \mathrm{BL}$ and in-plane remanent states (b), (d), (f), (h), and (j), respectively.

expected. In the sample with very weak out-of-plane anisotropy $(25 \mathrm{BL})$ the oscillations of the magnetization vector almost close the magnetic flux inside the sample, whereas for the larger samples the magnetic contrast in the MFM images (see Fig. 4) increases with the thickness of the samples.

In Fig. 5 we show the average stripe domain period extracted from 2DFTs as a function of BL number in the virgin and in-plane remanent states. The average domain period increases with increasing thickness and exhibits a nonlinear 


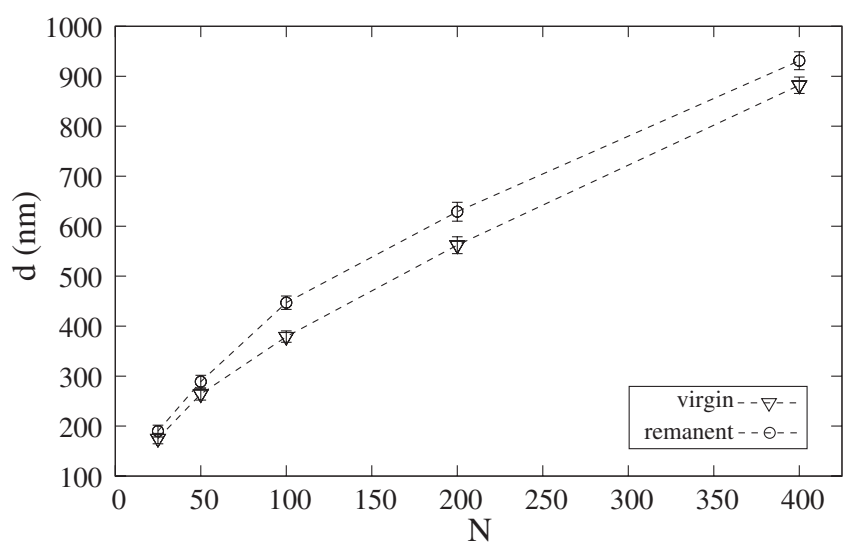

FIG. 5. The dependence of the stripe domain period on the number of bilayers for the $\mathrm{Fe}_{81} \mathrm{Ni}_{19} / \mathrm{Co}$ superlattices in the virgin and in-plane remanent states.

dependence. For small BL number, the period increases slightly more rapidly than for large $N$. The observed difference in domain period between virgin and in-plane remanent states is connected to different magnetization structures: The virgin state is expected to consist of easy axis domains which are separated by $180^{\circ}$ walls, whereas in the remanent state the magnetization is oriented at oblique angles with respect to the film surface. This affects the relative importance of the competing energy terms [cf. Eq. (2) in Sec. III A], giving rise to different domain periods.

\section{THEORY}

The general difficulty one is faced with in calculating the ground state of a magnetic system is the fact that the total energy of the system is a functional of the magnetization distribution $\mathbf{M}(\mathbf{r})$ and that an analytical expression for this functional is usually not available. The reason is that the energy term due to the magnetic stray field is in general not explicitly known and needs to be obtained by solving Maxwell's equations. The magnetization distribution thus needs to be determined by a self-consistent procedure. The magnetization distribution is in general a complex function in space, the form of which is usually not known a priori. However, there exist certain limiting cases, where it can be parameterized. In these rare cases the stray field energy can usually be calculated analytically as a function of certain parameters. The ground-state energy and the ground-state magnetization distribution are then obtained by minimization of the total energy with respect to these parameters.

In the general case and for general sample geometries the total-energy functional may be evaluated numerically using the finite element method (FEM) for the discretization of the problem and the ground state is found by performing a conjugate gradient search for the energy minimum. ${ }^{34}$ Another possibility is to calculate the dynamics by numerically integrating the corresponding Landau-Lifshitz-Gilbert equation directly which yields the ground state after a relaxation process. ${ }^{35}$ However, these approaches are computationally feasible only for relatively small systems and therefore cannot be applied to the samples under consideration.
In Sec. III A we approach the problem by assuming a certain magnetization structure and parameterizing it using the tilt angle of the magnetization with respect to the surface normal and the domain period as parameters. This allows to calculate the interaction of the magnetization with the stray field analytically. The resulting equations give an intuitive understanding of the underlying mechanisms and give qualitatively correct predictions. However, the model fails quantitatively. In Sec. III B we therefore pass over to a more elaborate model by considering the solutions of a linearized Landau-Lifshitz equation, which is possible due to the relatively simple geometry of the samples. The influence of the stray field is included by solving this equation simultaneously with Maxwell's equations for the stray field.

\section{A. Parameterized magnetization}

In a first attempt to describe the experimental results, we assume a specific (static) magnetization structure, not accounting for the possible existence of closure domains in these samples. This model has been applied to a different $\mathrm{Fe}_{81} \mathrm{Ni}_{19} / \mathrm{Co}(100)$ sample in a previous paper with reasonable results. ${ }^{26}$ We only briefly discuss the model here. A detailed description of the model was presented in Ref. 26.

In the case of sufficiently large out-of-plane anisotropy (of the order of the demagnetizing energy constant), the multilayer can be considered to be essentially magnetized parallel to a plane defined by the vertical axis and the direction of the external in-plane magnetic field.

Hence, the samples consisting of $N$ bilayers of thickness $D=t+s$ are assumed to exhibit a stripe domain structure with perpendicular magnetization components $\pm M_{s} \cos \theta$ alternating in adjacent domains and uniform in-plane magnetization $M_{s} \sin \theta$.

The angle $\theta$ is assumed to be the same for the $\mathrm{Fe}_{81} \mathrm{Ni}_{19}$ (index 1) and Co (index 2) layers with thicknesses $t_{1}$ and $t_{2}$. The total energy (calculated per unit volume and normalized to the average demagnetizing energy constant $K_{d}$ $\left.=4 \pi\left[\left(M_{s}^{(1)}\right)^{2} t_{1}+\left(M_{s}^{(2)}\right)^{2} t_{2}\right] / D\right)$ is the sum of the anisotropy-, demagnetizing-, and domain-wall energy terms, i.e.,

$$
e=e_{a}+e_{d}+e_{w} .
$$

We assume uniaxial perpendicular anisotropy: $e_{a}=\beta \sin ^{2} \theta$, where $\beta=K_{u} / K_{d}$ is identical to the quality factor $Q$. Effects of weaker superimposed (e.g., cubic) anisotropies are neglected. The stray field energy may be obtained by solving the corresponding Maxwell equations in the continuum approximation and can be written as $e_{d}=f(d, N) \cos ^{2} \theta$, where $f(d, N)$ plays the role of a $d$-dependent demagnetizing factor (for the explicit form of this expression and the dependence on the FeNi and Co-layer thicknesses, see Ref. 26). The demagnetizing energy $e_{d}(d, N)=f(d, N)$ for perpendicular magnetization and a fixed domain period $d$ is plotted in Fig. 6 as a function of the BL number. The energy decreases with increasing $N$ since the magnetostatic interaction between layers reduces the demagnetizing energy. However, adding a layer to a large stack of layers has a smaller effect: The average distance between layers increases with $N$ and their interaction becomes weaker with increasing distance. Hence 


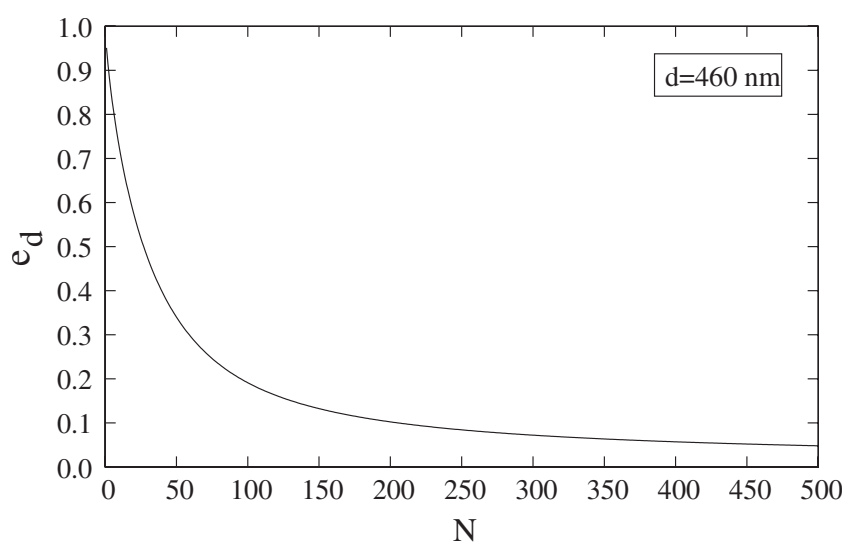

FIG. 6. Demagnetizing energy $e_{d}(d, N)=f(d, N)$ as a function of $\mathrm{BL}$ number for perpendicular magnetization and a fixed domain period $d=460 \mathrm{~nm}$.

the demagnetizing energy becomes independent of $N$ in the limit of large layer numbers. Since the demagnetizing energy contribution to Eq. (2) is the only one which explicitly depends on $N$, this effect explains why the experimental saturation field saturates for the thickest samples.

The remaining energy term, the domain-wall energy, can be expressed as $e_{w}=2 \tau(\theta) / d$, where $\tau(\theta)=\sigma_{w}(\theta) / K_{d}$ is the domain-wall tension, which can be approximated analytically using the simple model of a one-dimensional Bloch wall. Energy minimization with respect to $d$ and $\theta$ yields two coupled implicit equations for the domain period and inplane magnetization $M_{\|} / M_{s}=\sin \theta$ as a function of $N$ :

$$
\begin{gathered}
d(N)=\frac{1}{\cos \theta}\left[2 \tau(\theta) /\left(\frac{\partial f(d, N)}{\partial d}\right)\right]^{1 / 2}, \\
\sin \theta(N)=-\left[\frac{1}{d \cos \theta}\left(\frac{\partial \tau}{\partial \theta}\right)\right] /[\beta-f(d, N)] .
\end{gathered}
$$

Note that in the above equation $\partial \tau / \partial \theta \leq 0$. From these equations one may immediately deduce that the domain-wall energy favors large domain periods and small out-of-plane magnetization $\theta$, as expected. Furthermore, the stray field energy tends to decrease the domain period and the out-ofplane component of the magnetization as it acts like a uniaxial in-plane anisotropy. This can also be seen by including the interaction with an applied field in the film plane, $e_{h}=-2 h m \sin \theta$ into the total energy, Eq. (2), and minimizing with respect to $\theta$. This yields

$$
h(N)=\frac{1}{m}\left\{[\beta-f(d, N)] \sin \theta+\frac{1}{d \cos \theta} \frac{\partial \tau}{\partial \theta}\right\} .
$$

Both the perpendicular anisotropy and the wall tension, which is proportional $\sqrt{A K_{u}}$, increase the saturation field. It should be noted, however, that a larger exchange effectively reduces the saturation field, although it increases the wall tension. The reason is that it causes the domain period to increase, which on the one hand reduces the wall energy term and on the other enhances the shape anisotropy which favors in-plane orientation.
In order to apply our model, we need the exchange and anisotropy constants which were not measured for the samples. In this model it is possible to assign effective constants $A, K_{u}$ for a bilayer, since the angle $\theta$ between the magnetization and the surface normal is assumed to be the same in all layers. The two parameters can now be determined from the conditions that they should reproduce the experimentally measured domain period $d_{r}$ and saturation field for the $25 \mathrm{BL}$ sample. As already mentioned, the estimated saturation fields do not provide quantitative estimates of the true saturation fields. However, here we assume that the $25 \mathrm{BL}$ sample is on the verge of the transition to the homogeneously magnetized state so that the saturation field is approximately zero. Using these conditions, we find the rather large values $A=1.53 \cdot 10^{-5} \mathrm{erg} / \mathrm{cm}$ and $K_{u}=8.72 \cdot 10^{6} \mathrm{erg} / \mathrm{cm}^{3}$.

The domain period in the remanent state and the in-plane remanence as calculated from Eqs. (3) and (4) as functions of $N$ are shown in Figs. 7(a) and 7(b). As required, the results coincide with the experimental results for $N=25$. The results are in qualitative agreement with the experiment: The domain period increases with $N$ and does not saturate, while the in-plane remanence strongly decreases for small BL numbers and saturates for the thickest samples. The saturation field obtained from Eq. (5) is plotted in Fig. 7(c). The saturation field obtained from these calculations turns out to be much larger than the experimental values given in Table I obtained by MOKE. We rescaled the experimental values for the saturation field by a factor of 9.0 in order to show that the theoretical results nevertheless capture the essential qualitative features: The saturation field strongly increases at small BL numbers and saturates for larger BL numbers.

It is clear from the figures, that this simple model does not provide any quantitative description of the experimental observations. The domain period is somewhat underestimated and more noticeable: the remanence drops down too fast as the BL number increases. The latter is an obvious consequence of the large anisotropy constant used in the calculation: In the $25 \mathrm{BL}$ sample, the demagnetizing energy balances the anisotropy $[\beta \approx f(d, N)]$, while for thicker samples the relative importance of the demagnetizing energy decreases. Since $\beta$ is large, the anisotropy rapidly turns the magnetization out of the plane, reducing the remanence. Although the saturation field is expected to be larger than the saturation field obtained MOKE measurements, the theoretical saturatino filed seems to be strongly overestimated. This is as well a consequence of the large anisotropy constant.

The physical and the main origin of this discrepancy can be attributed to the presence of (partial) flux-closure domains in the sample. These are not taken into account in this simple approach. For a given period $d$, the formation of partial fluxclosure domains will lead to a smaller $f(d, N)$ term corresponding to a smaller shape anisotropy and consequently to a smaller value of $\beta$ when fitting the parameters. Another source of the discrepancy could be the simple model of the domain wall which is probably not a good approximation in the presence of such domains.

To conclude, the observations can be qualitatively explained as follows: The demagnetizing energy decreases with increasing BL number due to the magnetostatic interaction between the layers. Thereby the perpendicular anisotropy 

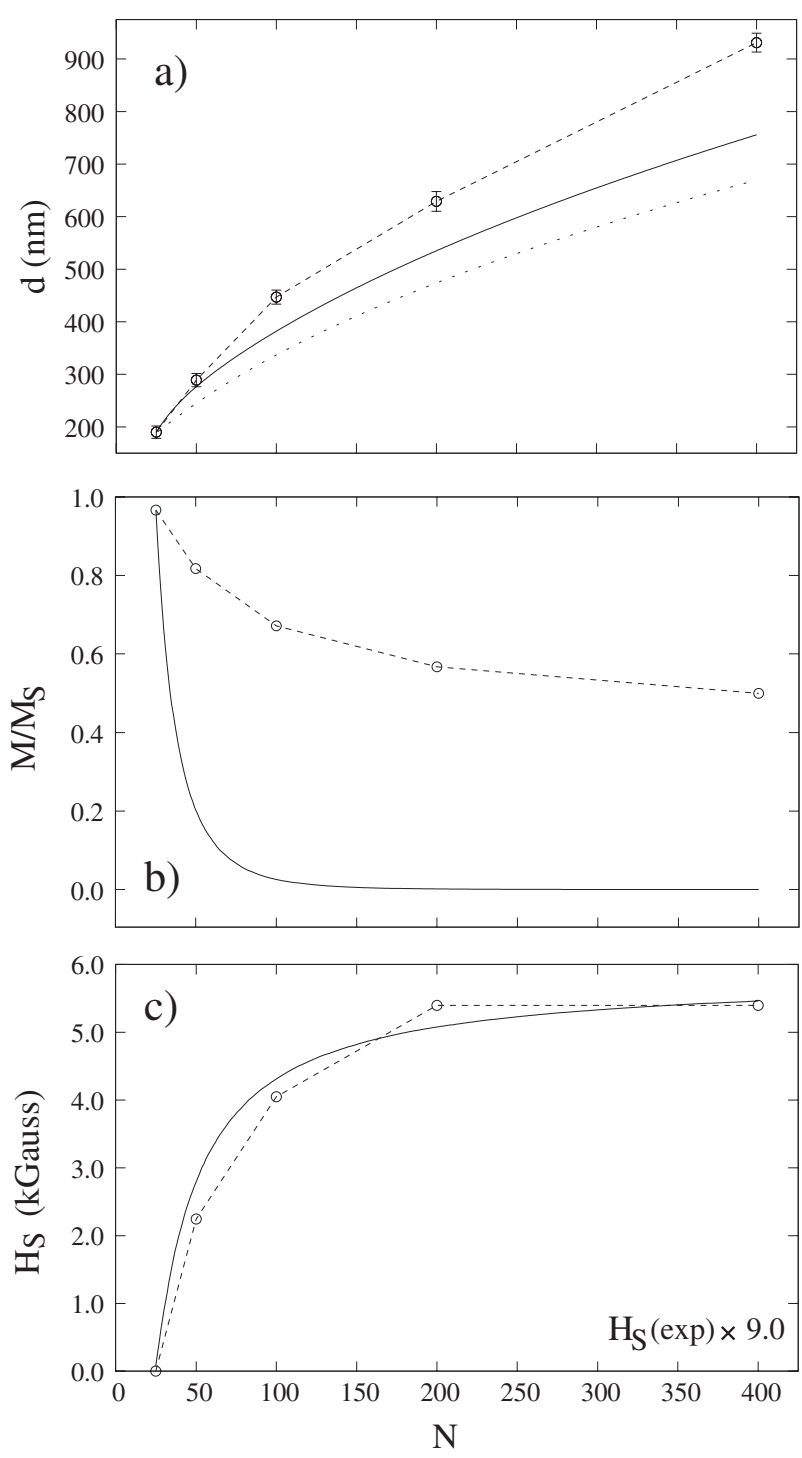

FIG. 7. Comparison between theoretical (solid lines) and experimental (dashed lines) results: (a) Domain period $d_{r}$ in the remanent state. The lower dotted curve shows the domain period at saturation. (b) In-plane remanent magnetization $M_{r} / M_{s}$ as a function of the bilayer number $N$. (c) Saturation field $H_{s}$ as a function of the BL number.

gains relative importance, causing the saturation field to increase as the magnetization turns out of the film plane. Consequently the wall energy also increases so that it becomes favorable to increase the mutual distance between walls and thus the domain period. The dependence of the demagnetizing energy on $N$ is reflected in the dependence of the magnetization and saturation field on this parameter.

Another qualitative conclusion that may be drawn from the calculated results is that the domain period at saturation is always smaller than in the remanent state and the difference between them grows with $N$. This is relevant for the interpretation of the results in Sec. III D.

\section{B. Landau-Lifshitz equation}

While being qualitatively correct, the results in Sec. III A show significant deviations from the experimental values. In order to obtain a quantitative description of the experimental observations, we attempt to solve the equation of motion for the samples. A similar method has been applied to obtain the critical field and critical thickness of single ferromagnetic layers, ${ }^{36,37}$ to calculate the spin-wave dispersion of magnetic films and multilayers ${ }^{38,39}$ and in the theory of ferromagnetic resonance..$^{40,41}$

We are mainly interested in the saturation field as a function of the film thickness, i.e., in the case where the sample is close to the phase transition from the state of homogeneous magnetization to the domain structure. Thus it may be assumed that the magnetization is mainly aligned along an inplane direction, parallel to an external field if present, with small components in the perpendicular directions. It is then possible to linearize the corresponding Landau-Lifshitz (LL) equation in these small components around the phasetransition point.

Starting point is the general energy functional for the ferromagnet

$$
W=\int d V\left\{\frac{A}{M^{2}}(\nabla \mathbf{M})^{2}+E_{a}[\mathbf{M}]-\mathbf{M} \cdot \mathbf{H}^{e}-\frac{1}{2} \mathbf{M} \cdot \mathbf{H}^{d}[\mathbf{M}]\right\},
$$

where the individual terms being the exchange-, anisotropy-, Zeeman, and demagnetizing field contributions (in this order).

As explained below, it is sufficient to consider the LL equation without damping, which has the form

$$
\frac{1}{\gamma} \frac{\partial \mathbf{M}}{\partial t}=\mathbf{M} \times \mathbf{H}^{\mathrm{eff}}
$$

and describes the precession of the magnetization in an effective field. As was shown by Landau and Lifshitz, ${ }^{42}$ the effective field can be obtained as the functional derivative of the energy functional with respect to the magnetization, which yields

$$
\mathbf{H}^{\mathrm{eff}}(\mathbf{r}, t)=-\frac{\delta W}{\delta \mathbf{M}(\mathbf{r}, \mathbf{t})}=\frac{2 A}{M^{2}} \Delta \mathbf{M}-\nabla_{\mathbf{M}} E_{a}+\mathbf{H}^{e}+\mathbf{H}^{d} .
$$

In order to fully take into account the influence of the stray field caused by the discontinuities of the magnetization at the surfaces and interfaces of the sample, one should solve this equation together with the Maxwell equations for the demagnetizing field, which may be written in magnetostatic approximation: ${ }^{43}$

$$
\nabla \times \mathbf{H}^{d}=0 \quad \nabla \cdot\left(\mathbf{H}^{d}+4 \pi \mathbf{M}\right)=0 .
$$

Using the fact that the field can be written as the gradient of a magnetic potential, $\mathbf{H}^{d}=-\nabla \phi$, the LL equation is then solved together with the scalar equation $4 \pi \nabla \mathbf{M}-\Delta \phi=0$ for the unknowns $\mathbf{M}$ and $\phi$.

The solutions for the magnetization and the demagnetizing field within each individual layer have to satisfy certain boundary conditions. For the field these are the standard conditions that the parallel component of $\mathbf{H}$ and the perpendicular component of $\mathbf{B}=\mathbf{H}+4 \pi \mathbf{M}$ should be continuous at the interface or surface. 
For the magnetization, the boundary condition for surfaces located at $z=d_{0}$ is the Rado-Wertman boundary condition $^{40,41,44}$

$$
\mathbf{M} \times\left(\nabla_{\mathbf{M}} E_{s}-\alpha \frac{\partial \mathbf{M}}{\partial n}\right)_{z=d_{0}}=0,
$$

where $E_{s}$ is the surface anisotropy energy functional, $\alpha$ $=2 A / M^{2}$ and $\partial / \partial n$ is the derivative with respect to the surface normal. At interfaces where an interlayer exchange coupling may be present, the appropriate boundary conditions are the Hoffmann boundary conditions. ${ }^{45}$ Introducing the layer type index $l$ and the layer index $i$, these conditions for the interface between layers $i$ and $i+1$ can be written as

$$
\begin{aligned}
& \mathbf{M}_{l} \times\left(\nabla_{\mathbf{M}_{l}} E_{\mathrm{int}}^{(l)}-\alpha_{l} \frac{\partial \mathbf{M}_{l}}{\partial n_{i}}\right)_{z=d_{i}}-\left.\alpha_{l l^{\prime}} \mathbf{M}_{l}\right|_{z=d_{i}} \\
& \times\left.\mathbf{M}_{l^{\prime}}\right|_{z=d_{i+1}}=0 \text {, } \\
& \mathbf{M}_{l^{\prime}} \times\left(\nabla_{\mathbf{M}_{l^{\prime}}} E_{\text {int }}^{\left(l^{\prime}\right)}-\alpha_{l^{\prime}} \frac{\partial \mathbf{M}_{l^{\prime}}}{\partial n_{i+1}}\right)_{z=d_{i+1}}-\left.\alpha_{l l^{\prime}} \mathbf{M}_{l^{\prime}}\right|_{z=d_{i+1}} \\
& \times\left.\mathbf{M}_{l}\right|_{z=d_{i}}=0 .
\end{aligned}
$$

Here, $\alpha_{l l^{\prime}}=2 A_{l l^{\prime}} / M_{l} M_{l^{\prime}}$ is the interlayer exchange constant $\alpha$ as before and $E_{\text {int }}$ is the interface anisotropy contribution. These boundary conditions allow us to take both surface and interface anisotropy contributions, as well as interlayer exchange contributions into account.

\section{Computational procedure}

The calculation of the saturation field is based on the following considerations: Since we consider a linearized equation, one may consider each of the Fourier components of the solution separately. These are proportional to $\exp [i(\kappa x$ $+\Omega t)]$, where $\Omega:=\omega /\left(\gamma M_{0}\right)$ is a normalized frequency and $\gamma$ is the gyromagnetic ratio. $\kappa:=k_{x} / s$ is a wave vector normalized to the exchange length of the first layer $s=\sqrt{\alpha}$. The $x$ direction is oriented in the film plane and perpendicular to the preferred direction of the magnetization. The LL equation is solved together with Maxwell's equations to obtain the spin-wave dispersion $\Omega(\kappa)$. The square of the frequency is allowed to take on both positive and negative values. For positive values, $\Omega$ is real and the solutions are proportional to $\exp ( \pm i|\Omega| t)$, i.e., these are oscillating solutions around the in-plane magnetization and hence are stable. For $\Omega^{2}$ negative, the frequency is purely imaginary and the solutions have the form $\exp ( \pm|\Omega| t)$. Thus there are solutions that grow exponentially in time. This is no contradiction since damping was not taken into account. These unstable solutions signal the departure from a homogeneous magnetization distribution and the presence of a domain structure in the sample.

If the external field is large enough, all branches of the dispersion are above zero and all solutions are stable, so that the sample is homogeneously magnetized in the film plane. If the field is lowered, the dispersion will shift downwards. The critical field $H_{c}$ which characterizes the phase transition is defined as the field where the minimum of the lowest branch of the dispersion crosses zero. We note that the thus defined critical field is not the same as the saturation field. At the critical field, the sample will not be entirely saturated at any finite temperature due to the excitation of spin waves (we postpone the discussion of temperature effects to the end of Sec. III D). However, keeping this subtlety in mind, we shall use the two terms synonymously in the following.

For fields lower than the critical field the sample will exhibit a domain structure. Since the critical field is characterized by the appearance of a single stable and static solution, it is sufficient to consider the LL equation without damping. The instability at the phase-transition point is characterized by a single wave vector $k_{x}$ due to a minimum in the dispersion curve. The minimum in the dispersion curve is due to the fact that for small wave vectors, the spin-wave energy can be decreased by increasing the wave vector due to the dipolar interaction. For large wave vectors, the spin waves are exchange dominated and the dispersion increases with the wave vector. The domain period at the phase transition is equal to the wavelength of the critical fluctuations and is thus directly related to the characteristic wave vector of the transition by $d=2 \pi / k_{x}$.

In order to obtain the critical field we thus calculated the spin-wave dispersion by varying the external field until the above mentioned condition for the critical field was met. The domain period at the phase transition was then obtained from the wave vector corresponding to the minimum of the dispersion curve.

In more detail, the calculation procedure is as follows: For a given wave vector $\kappa$, the linearized LL equation and Maxwell's equations are solved individually for each layer for different values of the frequency $\Omega$. The solutions are inserted into the boundary conditions which form a homogeneous linear system. The dispersion $\Omega(\kappa)$ is defined by those values of the frequency for which the system of boundary conditions has a solution, or equivalently, for which the corresponding boundary-condition matrix $A(\kappa, \Omega)$ is singular. Finding these values is the computationally expensive and numerically difficult part of the calculation. Various numerical techniques are available to achieve this. A singular matrix is either signaled by one or more eigenvalues being equal to zero or a vanishing singular value obtained by singular value decomposition. However, as has already been noted in Ref. 39 , the computation of the roots of the secular equation is numerically difficult, especially for large samples and correspondingly large matrices. For numerical stability, it turns out to be crucial to calculate the Fourier components of the magnetization and the potential using the analytical expressions for the Fourier components, Eq. (B1). To improve stability, we rescaled the wave vectors by the exchange length so that all quantities that enter the boundary-condition matrix are of the order of unity. We found that the most stable procedure to locate the roots is to calculate the function $f_{\kappa}\left(\Omega^{2}\right)$ defined by $f_{\kappa}\left(\Omega^{2}\right):=\log |\operatorname{det}[A(\kappa, \Omega)]|$. At the roots this function exhibits extremely sharp peaks, as illustrated in Fig. 8. Due to the narrowness of these peaks and a finite numerical accuracy, $f$ does not fall down to $-\infty$ at the desired roots but rather displays minima at these positions. These minima where located by calculating the cubic interpolating spline of $f$ and detecting the peaks in the first (or second) derivative of 


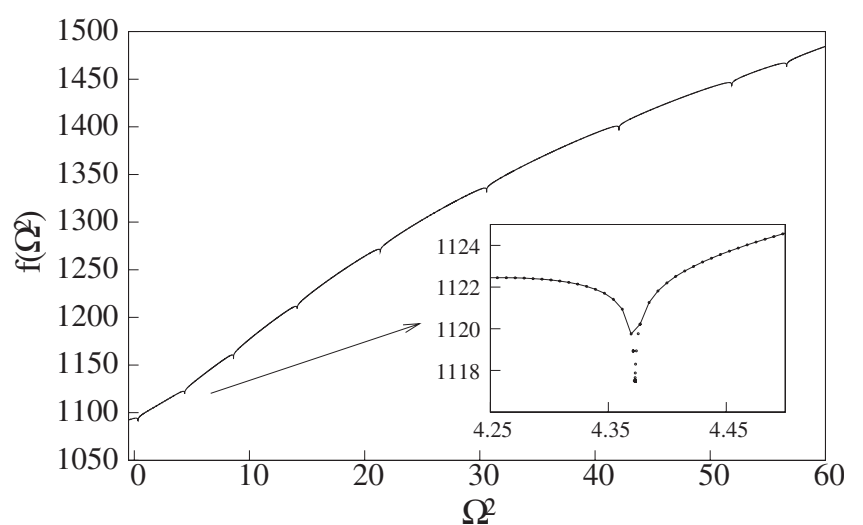

FIG. 8. Typical form of the function $f\left(\Omega^{2}\right)$ for the $25 \mathrm{BL}$ sample. The inset shows one of the minima with additional points obtained by a refinement using a bracketing algorithm with tolerance equal to the machine accuracy. For the $400 \mathrm{BL}$ sample the minima are not visible on this scale.

the spline. Since the boundary-condition matrix is banded with a full bandwidth of 17 elements, we used efficient bandmatrix algorithms for the LU factorization of the matrices with sizes up to $6000 \times 6000$.

The fact that $f$ displays minima instead of divergencies brings in the additional complication that zeros of $f$ cannot numerically be distinguished from minima. Therefore, nonphysical branches may appear in the final dispersion curve, as can be seen, e.g., in Fig. 12. However, these can be distinguished unambiguously from the real physical solutions using different criteria. For example, we found branches that are completely independent of the external field and would correspond to unstable solutions far beyond the saturation field. Such branches can also be ruled out by calculating the dispersion analytically by formulas that exist for certain limiting cases. ${ }^{36}$ Another branch that appeared in the calculations did not appear when the dispersion was calculated using two Fourier components for the magnetic field $h_{x}$ and $h_{z}$ instead of one for the potential $\varphi$ while the rest of the dispersion curve remained unchanged. Finally some points can be ruled out by directly examining $f\left(\Omega^{2}\right)$ because they correspond to broad local minima in this function. A brief deri- vation of the relevant equations in the form we use them is given in Appendix B.

\section{Calculations and results}

For the calculations, a number of parameters are needed. In order to reduce the number of free parameters, we performed first-principles calculations. The computations were performed for bulk FeNi and Co in an ideal structure. The chemical disorder was treated within the coherent-potential approximation. From these calculations, we extracted the saturation magnetization and the spin-wave stiffness constants $D$. The details of the computation of the stiffness constants from exchange parameters are presented in Appendix A. Corresponding results are given in Table II.

For the calculation of the saturation fields we neglect surface anisotropy contributions in the following since these should not essentially effect the behavior at the transition if they are not exceptionally large. The two surfaces only amount to a fraction as small as 0.04 with respect to the number of interfaces already for the smallest (25 BL) sample, which yields a corresponding contribution to the total anisotropy energy density. We checked numerically that indeed the surface anisotropy contributions are irrelevant for the calculation of the saturation fields. We further neglect the cubic anisotropy contribution in the following.

Under these assumptions, four constants remain to be determined, namely the bulk anisotropy for the two layer types, the interface anisotropy constant $K_{i}$, and the interlayer exchange $A_{\text {int }}$. In a previous study, ${ }^{28}$ it was found that in these multilayers the $\mathrm{Fe}_{81} \mathrm{Ni}_{19}$ layers are slightly expanded and the Co layers are slightly compressed along the $c$ axis. The inplane lattice parameter was found to be the same for both layers and equal to $2.84 \AA$. For $\mathrm{Fe}_{81} \mathrm{Ni}_{19}$ the out-of-plane lattice parameter was determined to be 2.89 and $2.82 \AA$ for Co. Since such distortions break the cubic symmetry, this gives rise to a uniaxial bulk anisotropy contribution. The corresponding anisotropy constants of order $n$ can be shown to be proportional to $(\xi / W)^{n}$, where $\xi$ is the strength of the spin-orbit coupling and $W$ is the width of the (usually narrow) $d$ band. ${ }^{50}$ Thus one expects a strong enhancement of the anisotropy which might be the source of the uniaxial bulk

TABLE II. Parameters for the solution of the Landau-Lifshitz equation. The three parameter sets correspond to three different choices for the bulk exchange constants, see text.

\begin{tabular}{|c|c|c|c|c|c|c|c|c|c|c|c|c|}
\hline & (a) & $\mathrm{Fe}_{81} \mathrm{Ni}_{19}$ & & Co & (b) & $\mathrm{Fe}_{81} \mathrm{Ni}_{19}$ & & Co & (c) & $\mathrm{Fe}_{81} \mathrm{Ni}_{19}$ & & Co \\
\hline $4 \pi M_{s}(\mathrm{kGauss})$ & & & & & & 21.90 & & 17.72 & & & & \\
\hline$g$ & & & & & & 1.918 & & $1.850^{\mathrm{a}}$ & & & & \\
\hline$D\left(\mathrm{meV} \AA^{2}\right)$ & & 203.38 & & 603.13 & & 203.38 & & $428.00^{\mathrm{b}}$ & & 203.38 & & $330.00^{\mathrm{c}}$ \\
\hline$A\left(10^{-6} \mathrm{erg} / \mathrm{cm}\right)$ & & 1.596 & & 3.971 & & 1.596 & & 2.818 & & 1.596 & & 2.173 \\
\hline$A_{\text {int }}\left(\mathrm{erg} / \mathrm{cm}^{2}\right)$ & & & 140 & & & & 45 & & & & 30 & \\
\hline$K_{i}\left(\mathrm{erg} / \mathrm{cm}^{2}\right)$ & & & 0.210 & & & & 0.250 & & & & 0.273 & \\
\hline$K_{u}\left(10^{6} \mathrm{erg} / \mathrm{cm}^{3}\right)$ & & & & & & -4.182 & & -0.899 & & & & \\
\hline$K_{i, \mathrm{eff}}\left(10^{6} \mathrm{erg} / \mathrm{cm}^{3}\right)$ & & & 1.228 & & & & 1.462 & & & & 1.596 & \\
\hline
\end{tabular}

a Reference 46.

${ }^{\mathrm{b}}$ Reference 47.

${ }^{\mathrm{c}}$ References 48 and 49. 
anisotropy in these samples. In a recent study, ${ }^{50}$ the magnetocrystalline anisotropy energy (MAE) was calculated for Fe, $\mathrm{Ni}$, and $\mathrm{Co}$ for structural distortions from first principles. Interestingly it was found that both for the compression of the $\mathrm{Co}(\mathrm{bcc})$ structure as well as expansion of $\mathrm{Fe}(\mathrm{bcc})$ and $\mathrm{Ni}$ (bcc), the MAE corresponds to an out-of-plane anisotropy. Here we use the values for $\mathrm{Co}$ and $\mathrm{Fe}$, since the latter dominates the composition (the values for $\mathrm{Ni}$ for the distortions found in our superlattices are comparable to those of $\mathrm{Fe}$ ). The values were obtained from the data shown in Fig. 1 of Ref. 50 and are given in Table II $\left(K_{u}\right)$. These calculations were done for the experimental equilibrium values for the volume of the respective elements in bulk. The deviation of the unit-cell volume of $\mathrm{FeNi}$ and $\mathrm{Co}$ in the multilayer from these values is less than $2 \%$ for Co and $1 \%$ from the value of pure Fe.

Now the interface anisotropy and the interlayer exchange remain to be determined. These are difficult to be calculated on an $a b$ initio level, since one explicitly needs to account for the broken symmetry at the interfaces, which makes the calculations computationally demanding. We therefore determine these parameters by treating them as fitting parameters. As in the previous section, we assume that the thinnest (25 $\mathrm{BL}$ ) sample is at the phase transition to the domain structure, so that the sample has critical thickness and the critical field is equal to zero. As conditions for the two parameters we require that the experimental saturation field and the domain period of the $25 \mathrm{BL}$ sample should be reproduced by the calculations.

This requirement leads to the parameter set given in Table II. It turns out that this condition cannot be fulfilled if we take $K_{i}=0$, since the $25 \mathrm{BL}$ turns out to be always unstable in zero field. In order to stabilize the $25 \mathrm{BL}$ sample, we thus employed an interface anisotropy of the easy-plane type $\left(K_{i}>0\right)$. Thus, we find evidence for the presence of such an anisotropy in these samples.

We further account for the possibility that the exchange constant for Co obtained from our $a b$ initio calculations might be overestimated. Indeed, experimental results ${ }^{47-49}$ indicate smaller values. To our knowledge, experimental values for the stiffness constant of FeNi in the particular composition used in this study are not available. We thus repeated the calculation by changing the bulk exchange of the Co-layer. We use two different experimental values for the spin-wave stiffness constant $D$, as indicated in Table II is related to the exchange stiffness constant $A$ by $D$ $=(2 A / M) g \mu_{B}$. As a result of using smaller bulk exchange, the interlayer exchange constant is smaller. We find similar and reasonable values for the interface anisotropy constants between 0.21 and $0.27 \mathrm{erg} / \mathrm{cm}^{2}$.

These anisotropy constants are surprisingly large. In order to compare them with the bulk anisotropy values, one may consider an average effective bulk anisotropy from the interfaces: $K_{i, \text { eff }}=\left(K_{i, \mathrm{Co}}+K_{i, \mathrm{FeNi}}\right) /\left(d_{\mathrm{Co}}+d_{\mathrm{FeNi}}\right)$. The numbers obtained are given in Table II. The values are of the same order of magnitude as the uniaxial out-of-plane anisotropy.

The results of these calculations are shown in Figs. 9 and 10. It can be seen that qualitatively the critical field behaves very similar for all three values of these constants. The curves very well resemble the experimental fact that the satu-

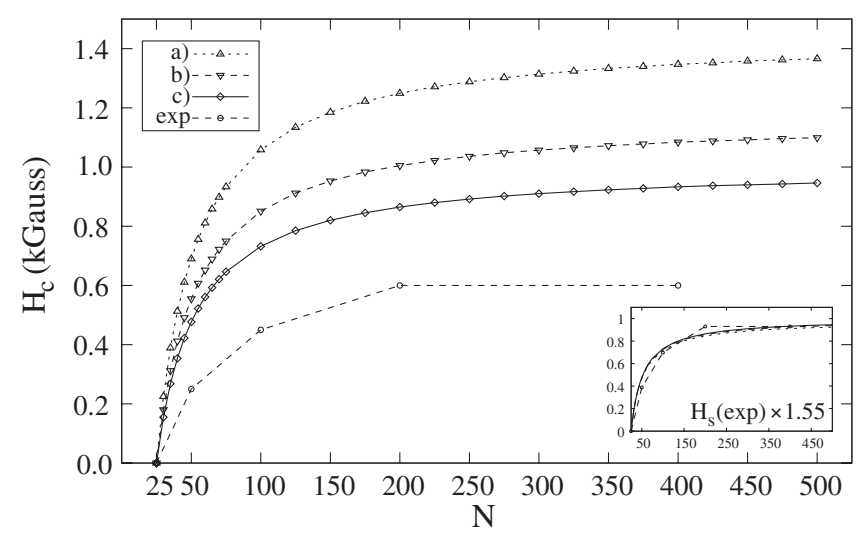

FIG. 9. Calculated critical field as a function of the bilayer number $N$ for the three different parameter sets of Table II. Experimental values for the saturation field estimated from the hysteresis curves in Fig. 1 are plotted with open circles.

ration field increases strongly for small $\mathrm{BL}$ numbers while it saturates for large BL numbers. In Fig. 10, one can see that the domain period at the transition is essentially the same for the three sets of parameters. Since no experimental data are available for the domain period at saturation for these samples, we cannot directly compare the results of the calculation with the experiment. However, as was shown in Sec. III A, one expects the domain period at saturation to be smaller than the domain period in zero field and this difference is expected to increase with the BL number. In Fig. 10, the results obviously follow this expectation.

We would like to point out one interesting feature of the saturation field curves in Fig. 9: Although the curves are quite different, we find that they can be brought to overlap by rescaling. This is depicted in the inset of Fig. 9. The relevant scaling factor turns out to be the ratio between the corresponding average bulk exchange constants $\left(A_{\mathrm{Co}}+A_{\mathrm{FeNi}}\right) / 2$ of the multilayers. This implies that the qualitative behavior is independent of the value of the exchange constants.

The reason for this simple scaling behavior is the rather strong exchange coupling between the layers. The interlayer exchange coupling is of the order of the strong-coupling

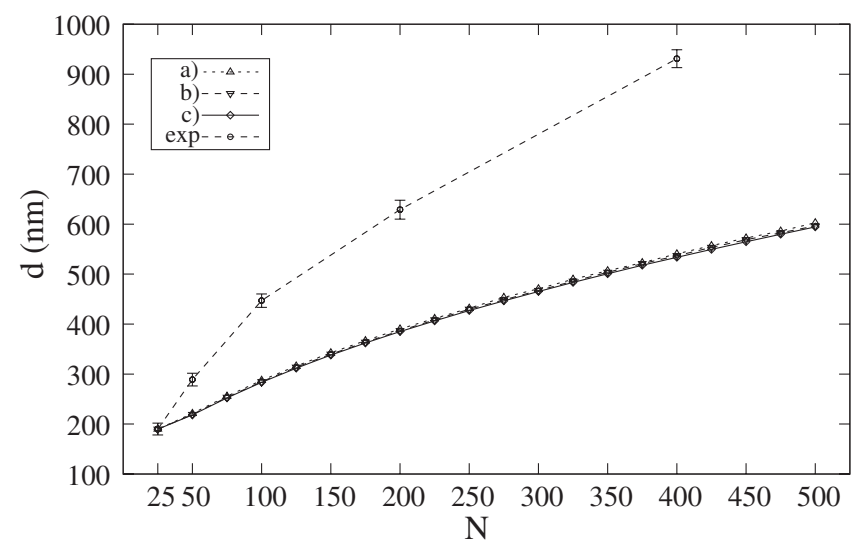

FIG. 10. Calculated domain period at the transition as a function of the bilayer number $N$ for the parameter sets in (a)-(c) in Table I. For comparison, the domain period of the samples in zero field is shown (dotted line). For explanation see text. 

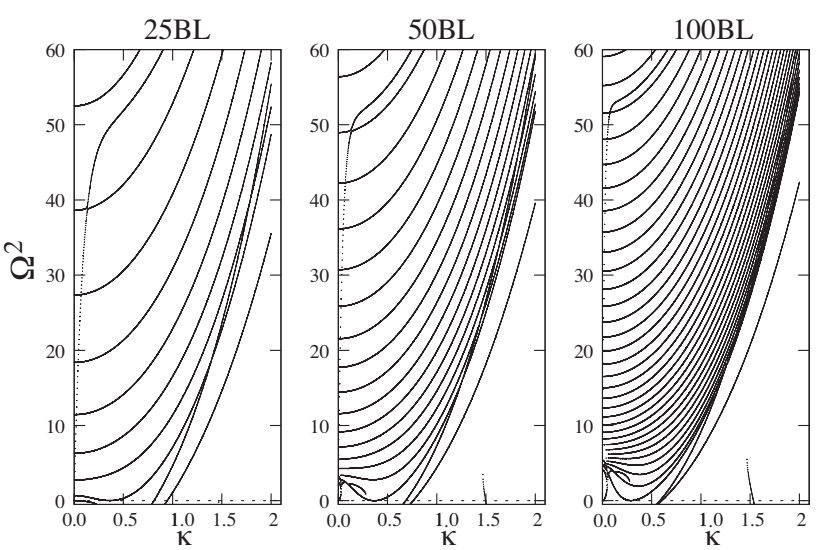

FIG. 11. Spin-wave dispersion for the 25, 50, and $100 \mathrm{BL}$ samples at their respective critical fields $(0,0.477$, and 0.732 kGauss) as a function of the normalized wave vector $\kappa=k s$, where $s=1.48 \cdot 10^{-6}$ is the exchange length. The range in $\kappa$ corresponds to a range of $1.35 \cdot 10^{6} \mathrm{~cm}^{-1}$ and the frequency range is approximately $28 \mathrm{GHz}$. The steep branch on the left is the surface mode.

limit, which for bcc and fcc lattices can be estimated as $A_{\text {int,sc }}=2 A / a$, where $a$ is the lattice constant. Due to the strong coupling, the multilayers act as an entity. Since the coupling is not perfect, the rescaled curves do not overlap completely (cf. inset of Fig. 9). As in the foregoing section, we also rescaled the experimental curves in order to show that we find a very good qualitative agreement (also shown in the inset of Fig. 9). Also in this case the theoretical result very well resembles the experimental result and the scaling factor seems more reasonable.

The conditions we used to adjust the interface anisotropy constant and the interlayer exchange are of course not unique. Another possibility is to require that the saturation fields for the 25 and $400 \mathrm{BL}$ samples should be reproduced. In this case we obtain very small values for the interlayer exchange constant. This results in a rather strong deviation from the experimental value of the domain period of the 25 BL sample. The reason is that MOKE indeed seems to underestimate the saturation field considerably and thus this condition should not be used.

For illustration, we show the spin-wave dispersions for the parameters given in Table II calculated at the respective critical fields in Figs. 11 and 12. In Fig. 12, one can see that for fields smaller than the critical field the dispersion shifts downwards, giving rise to unstable solutions indicating the presence of a domain structure.

We further considered the case where no interface anisotropy contributions are present. The mode of calculation is similar to that described in the foregoing, but now the interlayer exchange and the bulk instead of the interface anisotropy constants were adjusted. Essentially the same values for the interlayer exchange constants are obtained this way. We assumed the same anisotropy constants for both types of layers. This is justified since we find that shifting the relative weight of the anisotropy contributions between the layers essentially does not effect the results. We find the values $K_{u}=-0.9566,-1.086$, and -1.311 (in $10^{6} \mathrm{erg} / \mathrm{cm}^{3}$ ) for the parameter sets (a), (b), and (c) in Table II (with $K_{i}=0$ ), respectively. These values are very close to the values one
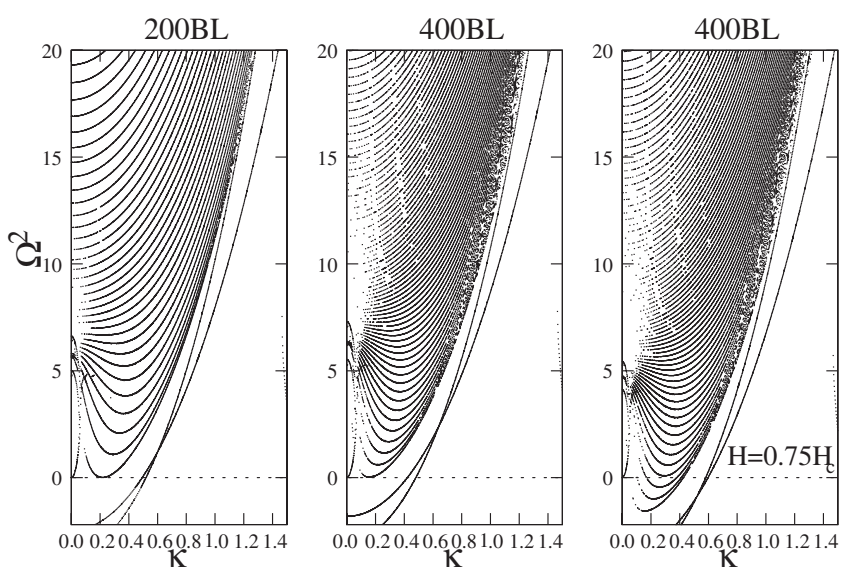

FIG. 12. Spin-wave dispersion for the 200 and 400 BL samples at their critical fields ( 0.865 and $0.933 \mathrm{kGauss})$ and somewhat below (right panel).

obtains if one adds the average bulk anisotropy constants and the effective interlayer anisotropy constants $\left(K_{u, \mathrm{Co}}\right.$ $+K_{u, \mathrm{FeNi}} / 2+K_{i, \text { eff. }}$. Approximating the interface anisotropy by an effective bulk anisotropy thus is a good approximation for calculating the saturation fields. The saturation field is determined by the average anisotropy energy density and is independent of the details of how the anisotropy is distributed. For these values of the parameters, the dependence of the critical field and the domain period are essentially the same as in Figs. 9 and 10 and therefore the results are not shown here.

In conclusion, we find that the results concerning the saturation field and domain period are essentially independent of the type of anisotropy and hence the question whether competing anisotropies are present in these samples cannot be definitely answered from this experimental data alone. However, we note that the conjecture of competing anisotropies is strongly supported by results from a subsequent study (to be presented elsewhere).

Unfortunately, no direct comparison with the experiment is available, since the saturation field obtained from the MOKE measurements is systematically underestimated and the domain period has not been measured at the saturation field. However, we believe that using the Landau-Lifshitz equation for the theoretical description of these samples provides a much better description of the multilayers. This is strongly supported by the fact that we obtain very reasonable results for our fitting parameters. Furthermore, we find very good qualitative agreement between theory and experiment concerning the saturation field, and also the domain period shows the expected behavior. The reason for a better agreement compared to the simple approach using a parameterized magnetization is that this approach implicitly accounts for the possibility of the formation of more complex domain structures like closure domains. The results obtained in Sec. III A clearly indicated that the shape anisotropy in the simple model was overestimated, which is expected in the presence of such domains.

We note that although temperature does not appear explicitly in this approach, temperature effects are implicitly accounted for through the parameters that enter the calculation. 
These should be regarded as effective parameters for the temperature at which the experiments were performed. In general, parameters such as the anisotropy or exchange constants are functions of temperature. For the case of a supercritical field, i.e., where all the branches of the dispersion are above zero energy, spin waves will be excited according to the Boltzmann factor $\exp \left(-E / k_{B} T\right)$ at any finite temperature. As a result, the magnetization approaches the maximum value asymptotically. Note that solutions with imaginary frequency are not physical since damping was not taken into account. Nevertheless they indicate the instability of the saturated in-plane magnetized state.

\section{CONCLUSIONS}

The nature of the magnetic anisotropy and the influence of the shape anisotropy on the evolution of the ground-state domain structure in bcc $\mathrm{Fe}_{81} \mathrm{Ni}_{19} / \mathrm{Co}(001)$ superlattices grown on $\mathrm{MgO}(001)$ were studied. At the thickness of about $85 \mathrm{~nm}$ the domains appear as stripe domains, typical for perpendicular anisotropy films, with a weak in-plane cubic anisotropy component which controls the stripe direction in the virgin state. The period of the domains was found to increase nonlinearly with the total thickness of the superlattice and a continuous rotation of the magnetization from inplane to out-of-plane for the thickness range from 85 to 1370 nm (25-400 BL) was observed.

Qualitative agreement was obtained by employing a simple model which assumes a simple magnetization structure. The model provides an intuitive understanding of the underlying physics. The dependence of the saturation field and domain period on the BL number has been explained as a consequence of the variation of the demagnetizing energy with the sample thickness. The results showed evidence for the presence of flux closure like domains in these samples.

Taking into consideration the existence of such domains by solving the Landau-Lifshitz equation, we considerably improved our theoretical model and reached fairly good agreement with the experimental data.

In the calculations, uniaxial bulk anisotropy constants obtained from first principles according to the experimentally determined lattice distortions were used. With these anisotropy constants, the results show evidence for the presence of an additional in-plane interface anisotropy, which partially compensates the bulk anisotropy. This is supported by the results from a subsequent study (this issue will be addressed in more detail elsewhere). Thus, the lattice distortions may be identified as the source of the perpendicular out-of-plane bulk anisotropy in these samples.

\section{ACKNOWLEDGMENTS}

We would like to thank T. Burkert for kindly providing the MAE data. Financial support from the Swedish Research Council (VR), Swedish Foundation for Strategic Research (SSF), and Göran Gustafsson Foundation is acknowledged. This work was supported by DFG Grant No. SFB 668 (Germany).

\section{APPENDIX A}

The electronic structure calculations were performed by means of the Green's function Korringa-Kohn-Rostoker (KKR) method. For the exchange-correlation functional the local-density approximation (LDA) has been used together with an spd basis. The chemical disorder was treated within the coherent-potential approximation (CPA). For the calculation of the stiffness constants $D$ we have adopted a realspace approach.

The exchange parameters were calculated using the theory of Lichtenstein et al., ${ }^{51}$ where the exchange interaction between two spins is calculated using a classical Heisenberg Hamiltonian employing the magnetic force theorem. The formula for the pair-exchange parameters reads

$$
J_{i j}=\frac{1}{4 \pi} \int^{E_{F}} d E \operatorname{Im} \operatorname{Tr}_{L}\left(\Delta_{i} T_{\uparrow}^{i j} \Delta_{j} T_{\downarrow}^{j i}\right),
$$

where $\Delta_{i}=t_{i \uparrow}^{-1}-t_{i \downarrow}^{-1}$ and $t$ being the on-site scattering matrices. $T$ is the scattering path operator which is related to the offdiagonal element of Green's function. $\operatorname{Tr}_{L}$ is the trace over the orbital indices of the scattering matrices. The integration over the full Brillouin zone was done using a total number of $10^{7} \mathbf{k}$ points.

The spin-wave stiffness constant $D$ can be expressed in terms of the pair-exchange parameters as

$$
D=\frac{2 \mu_{B}}{3 M} \sum_{j} J_{0 j} R_{0 j}^{2},
$$

where $M$ is the magnetic moment. The summation is over all sites $R_{0 j}$ but in practice it is performed up to a maximal value $R_{\max }$. We have considered pair-exchange parameters up to 102 shells $\left(R_{\max } \sim 8.6 a, a\right.$ being the lattice parameter). The sum in Eq. (A2) converges very poorly because of the oscillating behavior of the exchange interactions. This difficulty is resolved by substituting the expression with an equivalent one that is numerically convergent:

$$
D=\lim _{\eta \rightarrow 0} D(\eta)
$$

where

$$
D(\eta)=\lim _{R_{\max } \rightarrow \infty} \frac{2 \mu_{B}}{3 M_{0}} \sum_{0<R_{0 j} \leq R_{\max }} J_{0 j} R_{0 j}^{2} \exp \left(-\eta R_{0 j} / a\right) .
$$

The parameter $\eta$ plays the role of a damping parameter and ensures convergence of the sum over $R_{0 j}$.

The quantity $D(\eta)$ is evaluated for a set of values $\eta$, and the limit $\eta=0$ is then determined by a quadratic extrapolation method. For more details we refer the reader to the paper of Pajda et al. ${ }^{52}$

\section{APPENDIX B}

In the following, we briefly summarize the essential steps in the derivation of the equations used in the calculation of the spin-wave dispersions. 
Our coordinate system is oriented such that the perpendicular direction is the $z$ direction, the $y$ direction is the stripe direction or the direction of the applied field, and the $x$ direction labels the propagation direction of the spin waves. The multilayers are assumed to be infinitely extended in the $x-y$ plane. Close to the phase transition the magnetization is mainly aligned along an in-plane direction, parallel to an external field if present, with small components in the perpendicular directions. Thus one may write

$$
\mathbf{M}(\mathbf{r}, t)=\mathbf{M}_{0}+M_{0} \mathbf{m}(\mathbf{r}, t) .
$$

With $\mathbf{M}_{0}=\left(0, M_{0}, 0\right)$, one has

$$
\mathbf{M}=M_{0}\left(m_{x}, 1+m_{y}, m_{z}\right),
$$

where by assumption $m_{x, z} \ll 1$ and $m_{y} \approx-\frac{1}{2}\left(m_{x}^{2}+m_{z}^{2}\right) \approx 0$ in first-order approximation. The external field is given by $\mathbf{H}^{e}$ $=\left(0, H_{y}^{e}, 0\right)$.

Inserting this into Eqs. (6) and (7) one finds after division by $M_{0}^{2}$ that up to first order in $m_{x}, m_{z}$

$$
\begin{gathered}
\frac{1}{\gamma M_{0}} \frac{\partial m_{x}}{\partial t}=\alpha \Delta m_{z}-h_{\alpha} m_{z}-\frac{\partial \varphi}{\partial z}-h m_{z}, \\
\frac{1}{\gamma M_{0}} \frac{\partial m_{z}}{\partial t}=h m_{x}-\alpha \Delta m_{x}+h_{\beta} m_{x}-\frac{\partial \varphi}{\partial x} .
\end{gathered}
$$

The equation for $m_{y}$ is identically fulfilled. Here the definitions $h:=H_{y}^{e} / M_{0}, \varphi:=\phi / M_{0}$, and $h_{\alpha, \beta}:=H_{\alpha, \beta} / M_{0}$ have been introduced. $H_{\alpha, \beta}$ are the anisotropy fields defined by

$$
H_{\alpha} m_{z}=\frac{1}{M_{0}} \frac{d E_{a}}{d m_{z}}, \quad H_{\beta} m_{x}=\frac{1}{M_{0}} \frac{d E_{a}}{d m_{x}} .
$$

For the case of uniaxial perpendicular anisotropy $E_{a, u}$ $=K_{u} m_{z}^{2}$, these are given by $H_{\alpha}=2 K / M_{0}$ and $H_{\beta}=0$. Now passing over to Fourier transforms (time and space coordinates are rescaled),

$$
\begin{aligned}
m_{x, z}(x, y, z, t)= & \int d \mathbf{k} \int d \Omega m_{x, z}\left(\kappa, q_{y}, k, \omega\right) \\
& \times \exp \left[i\left(\kappa x+q_{y} y+k z+\Omega t\right)\right], \\
\varphi(x, y, z, t)= & \int d \mathbf{k} \int d \Omega \varphi\left(\kappa, q_{y}, k, \omega\right) \\
& \times \exp \left[i\left(\kappa x+q_{y} y+k z+\Omega t\right)\right],
\end{aligned}
$$

and introducing $\Omega:=\omega /\left(\gamma M_{0}\right)$ and $q^{2}=\kappa^{2}+q_{y}^{2}+k^{2}$ one may write the equations together with the Maxwell equation in the form

$$
\begin{gathered}
i \Omega m_{x}+\left(\alpha q^{2}+h_{\alpha}+h\right) m_{z}+i k \varphi=0, \\
\left(\alpha q^{2}+h_{\beta}+h\right) m_{x}-i \Omega m_{z}+i \kappa \varphi=0, \\
4 \pi i \kappa m_{x}+4 \pi i k m_{z}+q^{2} \varphi=0,
\end{gathered}
$$

which is a homogeneous linear system in the Fourier components $m_{x}, m_{z}$, and $\varphi$. The wave vectors are of order $10^{6}$ while the exchange constant is of the order $10^{-12}$. It is ad- vantageous to rescale the wave vectors by the exchange length $s=\sqrt{\alpha}$, which is of order $10^{-6}$. Introducing the rescaled quantities $\widetilde{\kappa}=\kappa s, \tilde{k}=k s, \tilde{q}=q s$, and $\tilde{\varphi}=\varphi / s$ one may write

$$
\left(\begin{array}{ccc}
i \Omega & \widetilde{q}^{2}+h_{\alpha}+h & i \tilde{k} \\
\tilde{q}^{2}+h_{\beta}+h & -i \Omega & i \widetilde{\kappa} \\
4 \pi i \widetilde{\kappa} & 4 \pi i \tilde{k} & \widetilde{q}^{2}
\end{array}\right) \cdot\left(\begin{array}{c}
m_{x} \\
m_{z} \\
\widetilde{\varphi}
\end{array}\right)=0 .
$$

This system has a nontrivial solution only if the determinant is equal to zero:

$$
\begin{gathered}
\Omega^{2} \widetilde{q}^{2}-\left(h+\widetilde{q}^{2}\right)\left[\widetilde{q}^{2}\left(h+h_{\alpha}+h_{\beta}+4 \pi+\widetilde{q}^{2}\right)-4 \pi \widetilde{q}_{y}^{2}\right] \\
-4 \pi\left(h_{\beta} \widetilde{k}^{2}+h_{\alpha} \widetilde{\kappa}^{2}\right)-h_{\alpha} h_{\beta} \widetilde{q}^{2}=0 .
\end{gathered}
$$

For given values of $\widetilde{\kappa}$ and $\tilde{q}_{y}$, this equation determines the dispersion $\Omega=\Omega(\tilde{k})$. The secular equation is of third order in $\widetilde{k}^{2}$ and therefore has six different solutions with respect to $\tilde{k}$ : $\tilde{k}_{1}, \tilde{k}_{3}, \tilde{k}_{5}$, and $\tilde{k}_{i+1}=-\tilde{k}_{i}$ for $i=1,3,5$. These are functions of $\widetilde{\kappa}, \tilde{q}_{y}$, and $\Omega$. Note that in our calculations we always take $\tilde{q}_{y}=0$ since the magnetization is assumed to be homogeneous along the $y$ direction. The three roots $\rho:=\widetilde{k}^{2}$ are the solutions to the third order polynomial equation

$$
\rho^{3}+a \rho^{2}+b \rho+c=0,
$$

with real coefficients. Introducing $\widetilde{q}_{\|}^{2}=\widetilde{\kappa}^{2}+\widetilde{q}_{y}^{2}$ the coefficients are

$$
\begin{gathered}
a=2 h+h_{\alpha}+h_{\beta}+4 \pi+3 \widetilde{q}_{\|}^{2}, \\
b=4 \pi \widetilde{\kappa}^{2}+\left(h+h_{\beta}+\widetilde{q}_{\|}^{2}\right)\left(4 \pi+h+h_{\alpha}+2 \widetilde{q}_{\|}^{2}\right) \\
+\widetilde{q}_{\|}^{2}\left(h+h_{\alpha}+\widetilde{q}_{\|}^{2}\right)-\Omega^{2}, \\
c=\left(h+h_{\alpha}+\widetilde{q}_{\|}^{2}\right)\left[4 \pi \widetilde{\kappa}^{2}+\widetilde{q}_{\|}^{2}\left(h+h_{\beta}+\widetilde{q}_{\|}^{2}\right)\right]-\widetilde{q}_{\|}^{2} \Omega^{2} .
\end{gathered}
$$

Therefore the solution to $m_{x}, m_{z}$, and $\phi$ in real space within the sample can be represented in the following form:

$$
\begin{aligned}
m_{x, z} & =\sum_{j=1}^{6} a_{j} m_{x, z}^{(j)}\left(\widetilde{\kappa}, \tilde{q}_{y}, \tilde{k}, \Omega\right) \exp \left(i \tilde{k}_{j} \frac{z}{s}\right), \\
\widetilde{\varphi} & =\sum_{j=1}^{6} a_{j} \widetilde{\varphi}_{j}\left(\widetilde{\kappa}, \widetilde{q}_{y}, \widetilde{k}, \Omega\right) \exp \left(i \widetilde{k}_{j} \frac{z}{s}\right),
\end{aligned}
$$

where the dependence on $x, y$, and $t$ has been absorbed into the quantities $a_{j}$. The Fourier components $m_{x, z}^{(j)}$ and $\widetilde{\varphi}_{j}$ can be calculated analytically using the system of linear equations and are determined up to a multiplicative factor:

$$
\begin{gathered}
m_{x, j}=4 \pi \widetilde{\kappa} \tilde{k}_{j}-i \Omega \widetilde{q}_{j}^{2}, \\
m_{z, j}=-\left[4 \pi \widetilde{\kappa}^{2}+\widetilde{q}_{j}^{2}\left(h+h_{\beta}+\widetilde{q}_{j}^{2}\right)\right], \\
\widetilde{\varphi}_{j}=4 \pi\left[i \widetilde{k}_{j}\left(h+h_{\beta}+\widetilde{q}_{j}^{2}\right)-\Omega \widetilde{\kappa}\right] .
\end{gathered}
$$

In the vacuum above or below the layers and in nonmagnetic layers, one has $m_{x, z} \equiv 0$. In this case the potential is a solution to the Laplace equation $\Delta \tilde{\varphi}=0$. In Fourier space, this becomes 


$$
\left(\widetilde{k}^{2}+\widetilde{q}_{y}^{2}+\widetilde{\kappa}^{2}\right) \widetilde{\varphi}=0,
$$

which has a nonvanishing solution for the potential only if $\widetilde{k}^{2}=-\widetilde{q}_{\|}^{2}$. Thus the solution for the potential in vacuum or nonmagnetic layers can be written as

$$
\widetilde{\varphi}=\widetilde{\varphi}_{+} \exp \left(\left|\widetilde{q}_{\|}\right| \frac{z}{S}\right)+\widetilde{\varphi}_{-} \exp \left(-\mid \widetilde{q}_{\|} \|_{S}\right) .
$$

Now the solutions are in principle known in all space. These solutions have to obey certain boundary conditions. For the potential one has to require $\widetilde{\varphi}_{+}=0$ at the top surface while $\widetilde{\varphi}_{-}=0$ at the bottom, since the magnetic field and the potential should vanish at infinity. The boundary conditions Eqs. (9) and (11) can now be expressed in terms of the solutions for the Fourier components Eq. (B1). In order to write down the equations in dimensionless form, the boundary conditions are divided by the exchange length of the type 1 layer, $s:=\sqrt{\alpha_{1}}$. Defining $\widetilde{h}_{\alpha ; s, i}^{(l)}=h_{\alpha ; s, i}^{(l)} / s, \widetilde{h}_{\beta ; s, i}^{(l)}=h_{\beta ; s, i}^{(l)} / s, s_{l}=\sqrt{\alpha_{l}} / s, \tilde{d}_{i}$ $=d_{i} / s$, and $\widetilde{q}_{\|}^{(l)}=\widetilde{q}_{\|} s_{l}$, the boundary conditions at the bottom surface $(\partial / \partial n=+\partial / \partial z)$ become

$$
\sum_{j=1}^{6} a_{j}^{(0)}\left\{4 \pi m_{z, j}^{(l)}-\left(i \widetilde{k}_{j}^{(l)}+\left|\widetilde{q}_{\|}^{(l)}\right|\right) \widetilde{\varphi}_{j}^{(l)}\right\} \exp \left(i \tilde{k}_{j}^{(l)} \frac{\widetilde{d}_{0}}{s_{l}}\right)=0,
$$

$$
\begin{aligned}
& \sum_{j=1}^{6} a_{j}^{(0)}\left\{\widetilde{h}_{\alpha, s}^{(l)}-i s_{l} \widetilde{k}_{j}^{(l)}\right\} m_{z, j}^{(l)} \exp \left(i \widetilde{k}_{j}^{(l)} \frac{\tilde{d}_{0}}{s_{l}}\right)=0, \\
& \sum_{j=1}^{6} a_{j}^{(0)}\left\{\widetilde{h}_{\beta, s}^{(l)}-i s_{l} \widetilde{k}_{j}^{(l)}\right\} m_{x, j}^{(l)} \exp \left(i \widetilde{k}_{j}^{(l)} \frac{\tilde{d}_{0}}{s_{l}}\right)=0 .
\end{aligned}
$$

The first condition is derived from Maxwell's equation and the last two are the Rado-Weertman boundary conditions. Similarly, at the top surface (layer $n$ ) one has $\partial / \partial n=-\partial / \partial z$ and the conditions read as

$$
\begin{gathered}
\sum_{j=1}^{6} a_{j}^{(n)}\left\{4 \pi m_{z, j}^{(l)}-\left(i \widetilde{k}_{j}^{(l)}-\left|\widetilde{q}_{\|}^{(l)}\right|\right) \widetilde{\varphi}_{j}^{(l)}\right\} \exp \left(i \widetilde{k}_{j}^{(l)} \frac{\widetilde{d}_{n}}{s_{l}}\right)=0, \\
\sum_{j=1}^{6} a_{j}^{(n)}\left\{\tilde{h}_{\alpha, s}^{(n)}+i s_{l} \widetilde{k}_{j}^{(l)}\right\} m_{z, j}^{(l)} \exp \left(i \widetilde{k}_{j}^{(l)} \frac{\widetilde{d}_{n}}{s_{l}}\right)=0, \\
\sum_{j=1}^{6} a_{j}^{(n)}\left\{\tilde{h}_{\beta, s}^{(n)}+i s_{l} \widetilde{k}_{j}^{(l)}\right\} m_{x, j}^{(l)} \exp \left(i \widetilde{k}_{j}^{(l)} \frac{\widetilde{d}_{n}}{s_{l}}\right)=0 .
\end{gathered}
$$

These conditions implicitly contain the fact that the potential outside the layers should continuously match the inside potential at the boundary. At magnetic/magnetic interfaces, the boundary conditions become (the lower layer has type index 1)

$$
\begin{aligned}
& \sum_{j=1}^{6} a_{j}^{(i)} \widetilde{\varphi}_{j}^{(l)} \exp \left(i \widetilde{k}_{j}^{(l)} \frac{\widetilde{d}_{i}}{s_{l}}\right)+\sum_{j=1}^{6} a_{j}^{(i+1)}(-1) \frac{m_{l^{\prime}}}{m_{l}} \frac{s_{l^{\prime}}}{s_{l}} \widetilde{\varphi}_{j}^{\left(l^{\prime}\right)} \exp \left(i \widetilde{k}_{j}^{\left(l^{\prime}\right)} \frac{\tilde{d}_{i}}{s_{l^{\prime}}}\right)=0, \\
& \sum_{j=1}^{6} a_{j}^{(i)}\left\{4 \pi m_{z, j}^{(l)}-i \widetilde{k}_{j}^{(l)} \widetilde{\varphi}_{j}^{(l)}\right\} \exp \left(i \widetilde{k}_{j}^{(l)} \frac{\tilde{d}_{i}}{s_{l}}\right)+\sum_{j=1}^{6} a_{j}^{(i+1)}(-1) \frac{m_{l^{\prime}}}{m_{l}}\left\{4 \pi m_{z, j}^{\left(l^{\prime}\right)}-i \widetilde{k}_{j}^{\left(l^{\prime}\right)} \widetilde{\varphi}_{j}^{\left(l^{\prime}\right)}\right\} \exp \left(i \widetilde{k}_{j}^{\left(l^{\prime}\right)} \frac{\widetilde{d}_{i}}{s_{l^{\prime}}}\right)=0, \\
& \sum_{j=1}^{6} a_{j}^{(i)}\left\{\widetilde{h}_{\alpha, i}^{(l)}+i s_{l} \widetilde{k}_{j}^{(l)}+\widetilde{\alpha}_{l l^{\prime}} \frac{m_{l^{\prime}}}{m_{l}}\right\} m_{z, j}^{(l)} \exp \left(i \widetilde{k}_{j}^{(l)} \frac{\widetilde{d}_{i}}{s_{l}}\right)+\sum_{j=1}^{6} a_{j}^{(i+1)}(-1) \widetilde{\alpha}_{l l^{\prime}} \frac{m_{l^{\prime}}}{m_{l}} m_{z, j}^{\left(l^{\prime}\right)} \exp \left(i \widetilde{k}_{j}^{\left(l^{\prime}\right)} \frac{\widetilde{d}_{i}}{s_{l^{\prime}}}\right)=0, \\
& \sum_{j=1}^{6} a_{j}^{(i)}\left\{\widetilde{h}_{\beta, i}^{(l)}+i \widetilde{k}_{l}^{(l)}+\widetilde{\alpha}_{l l^{\prime}} \frac{m_{l^{\prime}}}{m_{l}}\right\} m_{x, j}^{(l)} \exp \left(i \widetilde{k}_{j}^{(l)} \frac{\widetilde{d}_{i}}{s_{l}}\right)+\sum_{j=1}^{6} a_{j}^{(i+1)}(-1) \widetilde{\alpha}_{l l^{\prime}} \frac{m_{l^{\prime}}}{m_{l}} m_{x, j}^{\left(l^{\prime}\right)} \exp \left(i \widetilde{k}_{j}^{\left(l^{\prime}\right)} \frac{\widetilde{d}_{i}}{s_{l^{\prime}}}\right)=0, \\
& \sum_{j=1}^{6} a_{j}^{(i)}\left\{-\widetilde{\alpha}_{l l^{\prime}} \frac{m_{l}}{m_{l^{\prime}}}\right\} m_{z, j}^{(l)} \exp \left(i \widetilde{k}_{j}^{(l)} \frac{\widetilde{d}_{i}}{s_{l}}\right)+\sum_{j=1}^{6} a_{j}^{(i+1)}\left\{\widetilde{h}_{\alpha, i}^{\left(l^{\prime}\right)}-i s_{l^{\prime}} \widetilde{k}_{j}^{\left(l^{\prime}\right)}+\widetilde{\alpha}_{l l^{\prime}} \frac{m_{l}}{m_{l^{\prime}}}\right\} m_{z, j}^{\left(l^{\prime}\right)} \exp \left(i \widetilde{k}_{j}^{\left(l^{\prime}\right)} \frac{\widetilde{d}_{i}}{s_{l^{\prime}}}\right)=0, \\
& \sum_{j=1}^{6} a_{j}^{(i)}\left\{-\widetilde{\alpha}_{l l^{\prime}} \frac{m_{l}}{m_{l^{\prime}}}\right\} m_{x, j}^{(l)} \exp \left(i \widetilde{k}_{j}^{(l)} \frac{\widetilde{d}_{i}}{s_{l}}\right)+\sum_{j=1}^{6} a_{j}^{(i+1)}\left\{\widetilde{h}_{\beta, i}^{\left(l^{\prime}\right)}-i s_{l^{\prime}} \widetilde{k}_{j}^{\left(l^{\prime}\right)}+\widetilde{\alpha}_{l l^{\prime}} \frac{m_{l}}{m_{l^{\prime}}}\right\} m_{x, j}^{\left(l^{\prime}\right)} \exp \left(i \widetilde{k}_{j}^{\left(l^{\prime}\right)} \frac{\tilde{d}_{i}}{s_{l^{\prime}}}\right)=0 .
\end{aligned}
$$

Here the first two equations are the boundary conditions obtained from Maxwell's equations and the last four equations are the Hoffmann boundary conditions. 
${ }^{1}$ S.-B. Choe and S.-C. Shin, Phys. Rev. B 59, 142 (1999).

${ }^{2}$ K.-O. Ng and D. Vanderbilt, Phys. Rev. B 52, 2177 (1995).

${ }^{3}$ J. P. Whitehead and K. De'Bell, J. Phys.: Condens. Matter 6, L731 (1994).

${ }^{4}$ M. Seul and D. Andelman, Science 267, 476 (1995).

${ }^{5}$ O. Donzelli, D. Palmeri, L. Musa, F. Casoli, F. Albertini, L. Pareti, and G. Turilli, J. Appl. Phys. 93, 9908 (2003).

${ }^{6}$ O. Hellwig, G. P. Denbeaux, J. B. Kortright, and E. E. Fullerton, Physica B (Amsterdam) 336, 136 (2003).

${ }^{7}$ C. Favieres, C. Aroca, M. C. Sanchez, and V. Madurga, J. Appl. Phys. 91, 9995 (2002)

${ }^{8}$ T. Onoue, T. Asahi, K. Kuramochi, J. Kawaji, T. Osaka, J. Ariake, K. Ouchi, G. Safran, and N. Yaguchi, J. Appl. Phys. 92, 4545 (2002)

${ }^{9}$ S. Hamada, N. Hosoito, T. Ono, and T. Shinjo, J. Magn. Magn. Mater. 198-199, 496 (1999).

${ }^{10}$ L. Belliard, J. Miltat, V. Kottler, V. Mathet, C. Chappert, and T. Valet, J. Appl. Phys. 81, 5315 (1997).

${ }^{11}$ J. R. Barnes, S. J. O'Shea, M. E. Welland, J.-Y. Kim, J. E. Evetts, and R. E. Somekh, J. Appl. Phys. 76, 2974 (1994).

${ }^{12}$ T. Garel and S. Doniach, Phys. Rev. B 26, 325 (1982).

${ }^{13}$ Y. Yafet and E. M. Gyorgy, Phys. Rev. B 38, 9145 (1988).

${ }^{14}$ B. Kaplan and G. A. Gehring, J. Magn. Magn. Mater. 128, 111 (1993).

${ }^{15}$ P. Molho, J. L. Porteseil, Y. Souche, J. Gouzerh, and J. C. S. Levy, J. Appl. Phys. 61, 4188 (1987).

${ }^{16}$ C. Kittel, Phys. Rev. 70, 965 (1946).

${ }^{17}$ G. Ausanio, V. Iannotti, L. Lanotte, M. Carbucicchio, and M. Rateo, J. Magn. Magn. Mater. 226-230, 1740 (2001).

${ }^{18}$ W. B. Zeper, F. J. A. M. Greidanus, P. F. Carcia, and C. R. Fincher, J. Appl. Phys. 65, 4971 (1989).

${ }^{19}$ L. Louail, K. Ounadjela, M. Hehn, K. Khodjaoui, M. Gester, H. Danan, and R. Stamps, J. Magn. Magn. Mater. 165, 387 (1997).

${ }^{20}$ S. Hamada, K. Himi, T. Okuno, and K. Takanashi, J. Magn. Magn. Mater. 240, 539 (2002).

${ }^{21}$ P. F. Carcia, A. D. Meinhaldt, and A. Suna, Appl. Phys. Lett. 47, 178 (1985)

${ }^{22}$ H. J. G. Draaisma, W. J. M. de Jonge, and F. J. A. den Broeder, J. Magn. Magn. Mater. 66, 351 (1987).

${ }^{23}$ S. Park, S. Lee, and C. M. Falco, J. Appl. Phys. 91, 8141 (2002).

${ }^{24}$ R. Zdyb and E. Bauer, Phys. Rev. B 67, 134420 (2003).

${ }^{25}$ C. Liu, E. R. Moog, and S. D. Bader, Phys. Rev. Lett. 60, 2422 (1988).

${ }^{26}$ R. Brucas, H. Hafermann, M. I. Katsnelson, I. L. Soroka, O. Eriksson, and B. Hjörvarsson, Phys. Rev. B 69, 064411 (2004).
${ }^{27}$ P. V. de Weijer and J. S. Williams, Philips J. Res. 47, 237 (1993).

${ }^{28}$ I. L. Soroka, R. Bručas, V. Stanciu, P. Nordblad, and B. Hjörvarsson, J. Magn. Magn. Mater. 277, 228 (2004).

${ }^{29}$ J. H. Wolfe, R. K. Kawakami, W. L. Ling, Z. Q. Qiu, R. Arias, and D. L. Mills, J. Magn. Magn. Mater. 232, 36 (2001).

${ }^{30}$ A. Berger, U. Linke, and H. P. Oepen, Phys. Rev. Lett. 68, 839 (1992).

${ }^{31}$ P. Krams, B. Hillebrands, G. Güntherodt, and H. P. Oepen, Phys. Rev. B 49, 3633 (1994).

${ }^{32}$ From our measurements, we cannot determine the sign of $K_{c}$, but the same argument holds for $K_{c}>0$. A Fe/Co bcc film has [111] easy axis and hence $K_{c}<0$.

${ }^{33}$ A. Hubert and R. Schäfer, Magnetic Domains (Springer, New York, 1998).

${ }^{34}$ See, e.g., W. Rave and A. Hubert, IEEE Trans. Magn. 36, 3886 (2000).

${ }^{35}$ A publicly available finite difference micromagnetic solver is the "object oriented micromagnetic framework" developed at NIST (http://math.nist.gov/oommf/).

${ }^{36}$ A. Patashinski and W. Wasilewski, Acta Physiol. Pol. 57, 789 (1980).

${ }^{37}$ W. Wasilewski, Acta Physiol. Pol. 60, 7 (1981).

${ }^{38}$ G. T. Rado and R. J. Hicken, J. Appl. Phys. 63, 3885 (1988).

${ }^{39}$ B. Hillebrands, Phys. Rev. B 41, 530 (1990).

${ }^{40}$ G. T. Rado, Phys. Rev. B 26, 295 (1982).

${ }^{41}$ G. T. Rado, Phys. Rev. B 32, 6061 (1985).

${ }^{42}$ L. D. Landau and E. M. Lifshitz, Sow. Phys. 8, 153 (1935).

${ }^{43}$ A. I. Akhiezer, V. G. Bar'yakhtar, and S. V. Peletminskii, Spin Waves (North-Holland, Amsterdam, 1968).

${ }^{44}$ G. Rado and J. Weertman, J. Phys. Chem. Solids 11, 315 (1959).

${ }^{45}$ F. Hoffmann, A. Stankoff, and H. Pascard, J. Appl. Phys. 41, 1022 (1970).

${ }^{46}$ G. G. Scott, Phys. Rev. 104, 1497 (1956).

${ }^{47}$ X. Liu, M. M. Steiner, R. Sooryakumar, G. A. Prinz, R. F. C. Farrow, and G. Harp, Phys. Rev. B 53, 12166 (1996).

${ }^{48}$ F. Schreiber and Z. Frait, Phys. Rev. B 54, 6473 (1996).

${ }^{49}$ J. M. Karanikas, R. Sooryakumar, G. A. Prinz, and B. T. Jonker, J. Appl. Phys. 69, 6120 (1991).

${ }^{50}$ T. Burkert, O. Eriksson, P. James, S. I. Simak, B. Johansson, and L. Nordström, Phys. Rev. B 69, 104426 (2004).

${ }^{51}$ A. I. Liechtenstein, M. I. Katsnelson, V. P. Antropov, and V. A. Gubanov, J. Magn. Magn. Mater. 67, 65 (1987).

${ }^{52}$ M. Pajda, J. Kudrnovský, I. Turek, V. Drchal, and P. Bruno, Phys. Rev. B 64, 174402 (2001). 